\title{
ON THE CHROMOSPHERIC ACTIVITY NATURE OF A LOW-MASS CLOSE BINARY: KIC 12004834
}

\author{
E. Yoldaş and H. A. Dal \\ Department of Astronomy and Space Sciences, University of Ege, Bornova, İzmir, Turkey. \\ Received June 10 2018; accepted January 172019
}

\begin{abstract}
We study the nature of the chromospheric activity of an eclipsing binary KIC 12004834, using Kepler data. We analyse the light curve of the system, the sinusoidal variations at out-of-eclipses and detected flare events. The secondary component's temperature is found to be $4001 \pm 11 \mathrm{~K}$, the mass ratio is $0.743 \pm 0.001$, and the orbital inclination is $75^{\circ} .89 \pm 0^{\circ} .03$. The analysis indicates a stellar spot effect on the variation. Moreover, the OPEA model has been derived over 149 flares. The saturation level called Plateau value, is found to be $2.093 \pm 0.236 \mathrm{~s}$. The flare number per hour (known as flare frequency $N_{1}$ ) is found to be $0.06644 h^{-1}$, while the flare-equivalent duration per hour (known as flare frequency $N_{2}$ ) is found to be 0.59 second/hour. According to these results, KIC 12004834 is a very low-mass close binary system with high level of flare activity.
\end{abstract}

\section{RESUMEN}

Estudiamos la actividad cromosférica de la binaria eclipsante KIC 12004834 utilizando datos de Kepler. Analizamos la curva de luz y la variación sinusoidal fuera de eclipse, y detectamos ráfagas. Encontramos que la temperatura de la secundaria es de $4001 \pm 11 \mathrm{~K}$, que el cociente de masas es $0.743 \pm 0.001$ y la inclinación orbital es $75^{\circ} .89 \pm 0^{\circ} .03$. El análisis indica que hay un efecto de manchas en la variabilidad. Obtenemos el modelo OPEA para 149 ráfagas. El nivel de saturación, llamado Plateau tiene un valor de $2.093 \pm 0.236 \mathrm{~s}$. El número de ráfagas por hora, conocido como la frecuencia de ráfagas $N_{1}$, es $0.06644 h^{-1}$, mientras que la duración de la emisión equivalente a las ráfagas, conocida como la frecuencia $N_{2}$ es $0.59 \mathrm{~s} / \mathrm{hora}$. De acuerdo con estos resultados, KIC 12004834 es una binaria cerrada, de muy baja masa, y con frecuentes ráfagas.

Key Words: binaries: eclipsing — methods: data analysis — stars: flare — stars: individual: KIC 12004834 - stars: low-mass - techniques: photometric

\section{INTRODUCTION}

The nature of a low-mass eclipsing binary KIC 12004834 with $14^{m} .7180$ in Kepler band is studied here. KIC 12004834, whose nature is different from the classical UV Ceti type stars of spectral type dMe due to its being a binary system, was observed by Watson (2006) for the first time. Magnitudes of the system were given as $J=12^{\mathrm{m}} .007$, $H=11^{\mathrm{m}} .407, K=11^{\mathrm{m}} .170$ by Cutri et al. (2003). Although there are no further studies in the literature, some estimated parameters of the system were given by Coughlin et al. (2011), using calibrations to derive the parameters. Taking $T_{\text {eff }}=3576 \mathrm{~K}$, the orbital inclination $(i)$ was found to be $72^{\circ} .47$, while the masses were found to be $M_{1}=0.48 M_{\odot}$ and $M_{2}=0.34 M_{\odot}$. In addition, the radii were computed as $R_{1}=0.48 R_{\odot}$ and $R_{2}=0.35 R_{\odot}$. Like Coughlin et al. (2011), taking $T_{\text {eff }}=3576 \mathrm{~K}$, Slawson et al. (2011) found $\log (g)$ as $4.217 \mathrm{~cm} / \mathrm{s}^{2}$. There are several approaches to obtain the temperatures of its components. Coughlin et al. (2011) gave the temperatures as $T_{1}=3620 \mathrm{~K}$ for the pri- 
mary and $T_{2}=3468 \mathrm{~K}$ for the secondary. Armstrong et al. (2014) gave $T_{1}=3511 \mathrm{~K}$ for the primary and $T_{2}=3512 \mathrm{~K}$ for the secondary.

KIC 12004834 was mentioned as a chromospherically active system for the first time by Debosscher et al. (2011). In addition, a dominant flare activity was also reported by Balona (2015). Considering the estimated parameters of the system; KIC 12004834 is a low-mass close binary with a chromospherically active component. This makes the system an important object in the astrophysical sense. This is because the system exhibits not only spots, but also flare activity. A flaring star being a component of an eclipsing binary system is a rare phenomenon among the UV Ceti type stars. However, the red dwarf abundance is about $65 \%$ in our Galaxy, and seventyfive percent of them exhibit flare activity (Rodonó 1986). Thus, almost half of the stars in our galaxy should exhibit flare activity. The number of eclipsing binaries with a flaring component is nowadays increasing, thanks to space missions such the Kepler and Corot satellites.

Because of its effects on stellar evolution, flare activity is very important in astrophysics in terms of its sources and mechanism. Although the first flare was observed on the solar surface by R. C. Carrington and R. Hodgson on September 1, 1859 (Carrington 1859; Hodgson 1859), there are still unsolved problems, for instance, the different mass loss rate seen among stars of different spectral types, the different flare energy levels detected for stars of different spectral types (Gershberg \& Shakhovskaya 1983; Haisch et al. 1991; Gershberg 2005; Benz 2008).

At this point, photometric data accumulated from the eclipsing binaries with a chromospherically active component can give some clues for these problems. Recently, several eclipsing binary stars, where one of the components is chromospherically active have been discovered by the Kepler Mission (Borucki et al. 2010; Koch et al. 2010; Caldwell et al. 2010). Most of them have an interesting nature. These chromospherically active components exhibit flare events and also rapidly evolving stellar spots (Balona 2015). Although the light variations due to the cool spots have remarkably small amplitudes, their shapes change over short time intervals, from one cycle to the next one (Yoldaş \& Dal 2016, 2017; Özdarcan et al. 2017).

In this study, the variations of the times of minima are analysed (see $\S 2.1$ ). The light curve of KIC 12004834 is studied $(\S 2.2)$ for the first time in the literature in order to find the physical properties of the components. Then, the flares occurring on the chromospherically active component are used to model the nature of the magnetic activity of the system as described in $\S 2.3$. The results obtained are given in $\S 3$, comparing the active component with its analogue discovered in the Kepler Mission.

\section{DATA AND ANALYSES}

The data analysed in this study are the detrended short cadence data from the Kepler Mission Database (Borucki et al. 2010; Koch et al. 2010; Caldwell et al. 2010; Slawson et al. 2011; Matijevič et al. 2012). In the analyses, the data of quarters Q10.1, Q10.2 and Q10.3 are used (Murphy 2012; Murphy et al. 2013), whose quality and sensitivity are the highest ones ever reached (Jenkins et al. 2010a,b).

After removing all the observations with large errors from the data, and using the ephemeris taken from the Kepler Mission database, the phases are computed for all data, and the obtained light curves are shown in Figure 1. Because of the study's format, the detrended short cadence data were used in the analysis instead of those of the long cadence. The data were arranged in suitable formats for different analyses, such as the light curve analysis and the flare event calculations.

\subsection{Orbital Period Variation}

The times of minima in the light curves were computed from short cadence data without any corrections. We used just short cadence data, because the system has a very short orbital period. The whole shape of the minima is not seen in the long cadence data. The minima times were computed with a script according to the method described by Kwee \& van Woerden (1956). The $(O-C)_{I}$ residuals were determined for each minimum time. Examining the times of minima indicated that some of them have very large errors. These errors are sometimes caused by scattered observations; while some of them are caused by the flare activity occurring during these minima. All the minima times with large errors were removed from the $(O-C)$ data. Finally, 688 minima times were determined in the analyses.

Using the epoch of 2455002.041 and the orbital period of 0.2623168 day given in the Kepler Eclipsing Binary Catalogue ${ }^{1}$ by Slawson et al. (2011), we computed the $(O-C)_{I}$ residuals. Then, using the Least-Squares method, we applied a linear correction to these residuals. The linear correction revealed that the $(O-C)_{I}$ residuals had a linear trend with

\footnotetext{
${ }^{1}$ http://keplerebs.villanova.edu/.
} 


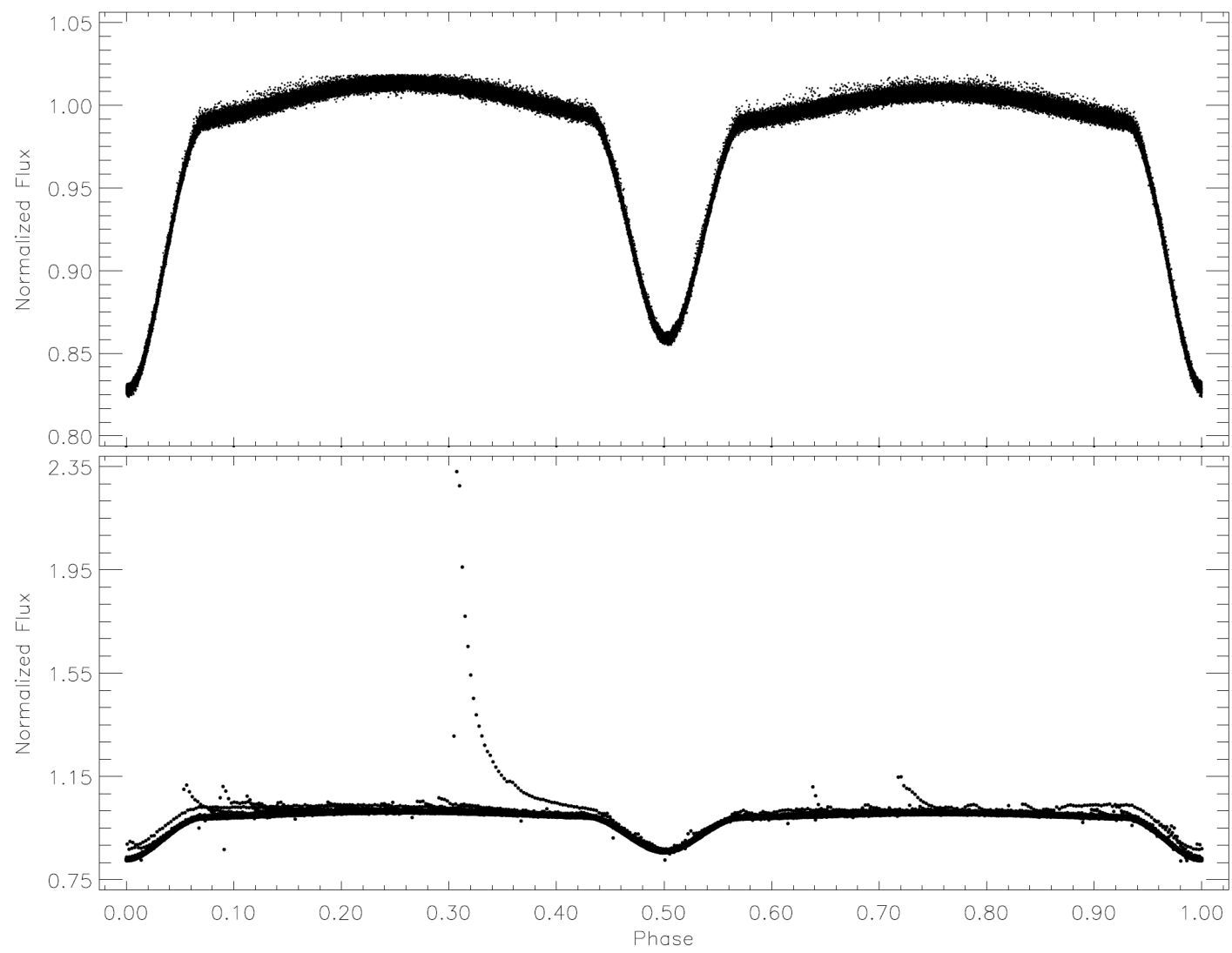

Fig. 1. Whole light curve of KIC 12004834 obtained from the data taken from the Kepler Mission database. In the bottom panel, the light curve is plotted along the orbital cycle with the flare activity, while it is plotted without the flare activity in the upper panel.

a small slope; the distribution of the $(O-C)_{I}$ residuals seems to be linear, needing just a zero point correction. After the linear correction, we obtained the new ephemerides given in equation (1) and the $(O-C)_{I I}$ residuals:

$J D($ Bary. $)=2455002.04164(14)+0^{d} .262317(1) \times E$.

The $(O-C)_{I}$ and $(O-C)_{I I}$ residuals are listed in Table 1. In the table, the minima times, cycles, the minima type, $(O-C)_{I}$ and $(O-C)_{I I}$ residuals are listed, respectively. An interesting variation is seen in the $(O-C)_{I I}$ residuals plotted versus time in Figure 2.

According to the studies of Tran et al. (2013) on contact binaries, if one of the components of an eclipsing binary system exhibits stellar spot activity on its surface, there must be a separation between the $(O-C)_{I I}$ residuals of the primary and secondary minima. Debosscher et al. (2011) and Balona (2015) mentioned that the system exhibits chromospheric activity. In the case of KIC 12004834 considered as a very close binary, we firstly examined whether there was any separation in the primary and secondary minimum $(O-C)_{I I}$ residuals. As shown in the upper panel of Figure 2, there is an evident separation between the primary and secondary minima residuals. Secondly, the analysis indicates that the best fit is derived by a linear function for the distribution of $(O-C)_{I}$. The obtained fit is shown in the lower panel of is Figure 2.

\subsection{Light Curve Analysis}

The light curve of KIC 12004834 was analyzed by the PHOEBE V.0.32 software (Prša \& Zwitter 2005) which depends on the 2003 version of the WilsonDevinney Code (Wilson \& Devinney 1971; Wilson 1990) to compute the physical parameters of each component. In the analyses, the averaged data were computed phase by phase with an interval of 0.001 to decrease the scattering. Although several modes 
TABLE 1

MINIMA TIMES AND THEIR RESIDUALS

\begin{tabular}{|c|c|c|c|c|c|c|c|c|c|}
\hline $\begin{array}{c}\text { BJD } \\
(+2450000) \\
\end{array}$ & $\mathrm{E}$ & Type & $\begin{array}{c}(O-C)_{I} \\
(\text { day })\end{array}$ & $\begin{array}{c}(O-C)_{I I} \\
\quad(\text { day })\end{array}$ & $\begin{array}{c}\text { BJD } \\
(+2450000) \\
\end{array}$ & $\mathrm{E}$ & Type & $\begin{array}{c}(O-C)_{I} \\
(\text { day })\end{array}$ & $\begin{array}{c}(O-C)_{I I} \\
(\text { day })\end{array}$ \\
\hline 5739.93853 & 2813.0 & I & 0.00037 & -0.00014 & 5833.06104 & 3168.0 & I & 0.00041 & -0.00008 \\
\hline 5740.20090 & 2814.0 & I & 0.00042 & -0.00009 & 5740.06987 & 2813.5 & II & 0.00055 & 0.00004 \\
\hline 5740.46318 & 2815.0 & I & 0.00039 & -0.00013 & 5740.33222 & 2814.5 & II & 0.00059 & 0.00007 \\
\hline 5740.72552 & 2816.0 & I & 0.00041 & -0.00010 & 5740.59460 & 2815.5 & II & 0.00065 & 0.00013 \\
\hline 5740.98782 & 2817.0 & I & 0.00040 & -0.00012 & 5740.85687 & 2816.5 & II & 0.00060 & 0.00008 \\
\hline 5741.25018 & 2818.0 & I & 0.00044 & -0.00008 & 5741.11948 & 2817.5 & II & 0.00090 & 0.00038 \\
\hline 5741.51253 & 2819.0 & I & 0.00047 & -0.00004 & 5741.38143 & 2818.5 & II & 0.00053 & 0.00001 \\
\hline 5741.77485 & 2820.0 & I & 0.00047 & -0.00004 & 5741.64370 & 2819.5 & II & 0.00049 & -0.00003 \\
\hline 5742.03709 & 2821.0 & I & 0.00040 & -0.00011 & 5741.90610 & 2820.5 & II & 0.00056 & 0.00005 \\
\hline 5742.29950 & 2822.0 & I & 0.00049 & -0.00002 & 5742.16839 & 2821.5 & II & 0.00054 & 0.00003 \\
\hline 5742.56182 & 2823.0 & I & 0.00049 & -0.00002 & 5742.43077 & 2822.5 & II & 0.00060 & 0.00008 \\
\hline 5742.82406 & 2824.0 & I & 0.00042 & -0.00009 & 5742.69298 & 2823.5 & II & 0.00049 & -0.00002 \\
\hline 5743.08650 & 2825.0 & I & 0.00054 & 0.00003 & 5742.95537 & 2824.5 & II & 0.00057 & 0.00006 \\
\hline 5743.34869 & 2826.0 & I & 0.00041 & -0.00010 & 5743.21765 & 2825.5 & II & 0.00053 & 0.00002 \\
\hline 5743.61107 & 2827.0 & I & 0.00048 & -0.00003 & 5743.47996 & 2826.5 & II & 0.00052 & 0.00001 \\
\hline 5743.87339 & 2828.0 & I & 0.00048 & -0.00003 & 5743.74229 & 2827.5 & II & 0.00054 & 0.00003 \\
\hline 5744.13561 & 2829.0 & I & 0.00038 & -0.00013 & 5744.00469 & 2828.5 & II & 0.00062 & 0.00011 \\
\hline 5744.39801 & 2830.0 & I & 0.00046 & -0.00005 & 5744.26691 & 2829.5 & II & 0.00052 & 0.00001 \\
\hline 5744.66025 & 2831.0 & I & 0.00038 & -0.00013 & 5744.52938 & 2830.5 & II & 0.00068 & 0.00016 \\
\hline 5744.92283 & 2832.0 & I & 0.00065 & 0.00014 & 5744.79153 & 2831.5 & II & 0.00051 & 0.00000 \\
\hline 5745.18490 & 2833.0 & I & 0.00041 & -0.00010 & 5745.05391 & 2832.5 & II & 0.00058 & 0.00006 \\
\hline 5745.44729 & 2834.0 & I & 0.00048 & -0.00003 & 5745.31632 & 2833.5 & II & 0.00067 & 0.00015 \\
\hline 5745.70963 & 2835.0 & I & 0.00050 & -0.00001 & 5745.57862 & 2834.5 & II & 0.00065 & 0.00013 \\
\hline 5745.97188 & 2836.0 & I & 0.00044 & -0.00007 & 5745.84093 & 2835.5 & II & 0.00064 & 0.00013 \\
\hline 5746.23418 & 2837.0 & I & 0.00041 & -0.00010 & 5746.10319 & 2836.5 & II & 0.00059 & 0.00008 \\
\hline 5746.49653 & 2838.0 & I & 0.00046 & -0.00006 & 5746.36550 & 2837.5 & II & 0.00058 & 0.00007 \\
\hline 5746.75883 & 2839.0 & I & 0.00044 & -0.00007 & 5746.62782 & 2838.5 & II & 0.00058 & 0.00007 \\
\hline 5747.02101 & 2840.0 & I & 0.00030 & -0.00021 & 5746.89012 & 2839.5 & II & 0.00057 & 0.00006 \\
\hline 5747.28347 & 2841.0 & I & 0.00044 & -0.00007 & 5747.15246 & 2840.5 & II & 0.00059 & 0.00008 \\
\hline 5747.54575 & 2842.0 & I & 0.00040 & -0.00011 & 5747.41478 & 2841.5 & II & 0.00060 & 0.00008 \\
\hline 5747.80808 & 2843.0 & I & 0.00042 & -0.00009 & 5747.67710 & 2842.5 & II & 0.00059 & 0.00008 \\
\hline 5748.07045 & 2844.0 & I & 0.00047 & -0.00005 & 5747.93950 & 2843.5 & II & 0.00068 & 0.00017 \\
\hline 5748.33278 & 2845.0 & I & 0.00048 & -0.00003 & 5748.20170 & 2844.5 & II & 0.00056 & 0.00005 \\
\hline 5748.59497 & 2846.0 & I & 0.00035 & -0.00016 & 5748.46406 & 2845.5 & II & 0.00061 & 0.00009 \\
\hline 5748.85738 & 2847.0 & I & 0.00045 & -0.00006 & 5748.72647 & 2846.5 & II & 0.00069 & 0.00018 \\
\hline 5749.11974 & 2848.0 & I & 0.00049 & -0.00002 & 5748.98876 & 2847.5 & II & 0.00067 & 0.00016 \\
\hline 5749.38192 & 2849.0 & I & 0.00036 & -0.00016 & 5749.25105 & 2848.5 & II & 0.00065 & 0.00013 \\
\hline 5749.64431 & 2850.0 & I & 0.00043 & -0.00008 & 5749.51321 & 2849.5 & II & 0.00049 & -0.00002 \\
\hline 5749.90661 & 2851.0 & I & 0.00042 & -0.00009 & 5749.77566 & 2850.5 & II & 0.00062 & 0.00011 \\
\hline 5750.16895 & 2852.0 & I & 0.00043 & -0.00008 & 5750.03785 & 2851.5 & II & 0.00050 & -0.00002 \\
\hline 5750.43123 & 2853.0 & I & 0.00040 & -0.00011 & 5750.30019 & 2852.5 & II & 0.00052 & 0.00001 \\
\hline 5750.69361 & 2854.0 & I & 0.00046 & -0.00005 & 5750.56269 & 2853.5 & II & 0.00070 & 0.00019 \\
\hline 5750.95592 & 2855.0 & I & 0.00046 & -0.00005 & 5750.82496 & 2854.5 & II & 0.00066 & 0.00014 \\
\hline 5751.21813 & 2856.0 & I & 0.00035 & -0.00016 & 5751.08720 & 2855.5 & II & 0.00058 & 0.00007 \\
\hline 5751.48043 & 2857.0 & I & 0.00033 & -0.00018 & 5751.34962 & 2856.5 & II & 0.00068 & 0.00017 \\
\hline 5751.74287 & 2858.0 & I & 0.00045 & -0.00006 & 5751.61171 & 2857.5 & II & 0.00046 & -0.00005 \\
\hline 5752.00513 & 2859.0 & I & 0.00039 & -0.00012 & 5751.87418 & 2858.5 & II & 0.00060 & 0.00009 \\
\hline 5752.26744 & 2860.0 & I & 0.00039 & -0.00012 & 5752.13672 & 2859.5 & II & 0.00083 & 0.00032 \\
\hline 5752.52964 & 2861.0 & I & 0.00027 & -0.00024 & 5752.39881 & 2860.5 & II & 0.00060 & 0.00009 \\
\hline 5752.79203 & 2862.0 & I & 0.00034 & -0.00017 & 5752.66107 & 2861.5 & II & 0.00055 & 0.00004 \\
\hline 5753.05428 & 2863.0 & I & 0.00028 & -0.00023 & 5752.92340 & 2862.5 & II & 0.00056 & 0.00005 \\
\hline 5753.31685 & 2864.0 & I & 0.00054 & 0.00003 & 5753.18572 & 2863.5 & II & 0.00056 & 0.00005 \\
\hline
\end{tabular}


TABLE 1 (CONTINUED)

\begin{tabular}{|c|c|c|c|c|c|c|c|c|c|}
\hline $\begin{array}{c}\text { BJD } \\
(+2450000)\end{array}$ & $\mathrm{E}$ & Type & $\begin{array}{c}(O-C)_{I} \\
\quad \text { (day) }\end{array}$ & $\begin{array}{c}(O-C)_{I I} \\
\quad(\text { day })\end{array}$ & $\begin{array}{c}\text { BJD } \\
(+2450000)\end{array}$ & $\mathrm{E}$ & Type & $\begin{array}{c}(O-C)_{I} \\
\quad(\text { day })\end{array}$ & $\begin{array}{c}(O-C)_{I I} \\
\quad \text { (day) }\end{array}$ \\
\hline 5753.57902 & 2865.0 & I & 0.00039 & -0.00012 & 5753.44802 & 2864.5 & II & 0.00054 & 0.00003 \\
\hline 5753.84128 & 2866.0 & I & 0.00033 & -0.00018 & 5753.71046 & 2865.5 & II & 0.00067 & 0.00016 \\
\hline 5754.10358 & 2867.0 & I & 0.00031 & -0.00020 & 5753.97273 & 2866.5 & II & 0.00063 & 0.00012 \\
\hline 5754.36600 & 2868.0 & I & 0.00042 & -0.00009 & 5754.23500 & 2867.5 & II & 0.00058 & 0.00007 \\
\hline 5754.62820 & 2869.0 & I & 0.00030 & -0.00021 & 5754.49742 & 2868.5 & II & 0.00068 & 0.00017 \\
\hline 5754.89082 & 2870.0 & I & 0.00060 & 0.00009 & 5754.75969 & 2869.5 & II & 0.00063 & 0.00012 \\
\hline 5755.15292 & 2871.0 & I & 0.00039 & -0.00012 & 5755.02200 & 2870.5 & II & 0.00062 & 0.00011 \\
\hline 5755.41537 & 2872.0 & I & 0.00053 & 0.00001 & 5755.28431 & 2871.5 & II & 0.00062 & 0.00011 \\
\hline 5755.67762 & 2873.0 & I & 0.00045 & -0.00006 & 5755.54643 & 2872.5 & II & 0.00042 & -0.00009 \\
\hline 5755.93995 & 2874.0 & I & 0.00047 & -0.00004 & 5755.80900 & 2873.5 & II & 0.00068 & 0.00017 \\
\hline 5756.20219 & 2875.0 & I & 0.00039 & -0.00013 & 5756.07126 & 2874.5 & II & 0.00062 & 0.00011 \\
\hline 5756.46450 & 2876.0 & I & 0.00038 & -0.00013 & 5756.33364 & 2875.5 & II & 0.00068 & 0.00017 \\
\hline 5756.72679 & 2877.0 & I & 0.00036 & -0.00015 & 5756.59594 & 2876.5 & II & 0.00066 & 0.00015 \\
\hline 5757.25137 & 2879.0 & I & 0.00030 & -0.00021 & 5756.85817 & 2877.5 & II & 0.00058 & 0.00007 \\
\hline 5757.51358 & 2880.0 & I & 0.00019 & -0.00032 & 5757.12057 & 2878.5 & II & 0.00066 & 0.00015 \\
\hline 5757.77606 & 2881.0 & I & 0.00036 & -0.00015 & 5757.38280 & 2879.5 & II & 0.00058 & 0.00007 \\
\hline 5758.03841 & 2882.0 & I & 0.00039 & -0.00012 & 5757.64519 & 2880.5 & II & 0.00065 & 0.00014 \\
\hline 5758.30065 & 2883.0 & I & 0.00032 & -0.00019 & 5757.90742 & 2881.5 & II & 0.00056 & 0.00005 \\
\hline 5758.56303 & 2884.0 & I & 0.00037 & -0.00014 & 5758.16977 & 2882.5 & II & 0.00060 & 0.00009 \\
\hline 5758.82529 & 2885.0 & I & 0.00032 & -0.00019 & 5758.43218 & 2883.5 & II & 0.00069 & 0.00018 \\
\hline 5759.08774 & 2886.0 & I & 0.00045 & -0.00006 & 5758.69452 & 2884.5 & II & 0.00071 & 0.00020 \\
\hline 5759.35011 & 2887.0 & I & 0.00051 & -0.00001 & 5758.95684 & 2885.5 & II & 0.00072 & 0.00021 \\
\hline 5759.61229 & 2888.0 & I & 0.00037 & -0.00014 & 5759.21913 & 2886.5 & II & 0.00068 & 0.00017 \\
\hline 5759.87448 & 2889.0 & I & 0.00025 & -0.00026 & 5759.48141 & 2887.5 & II & 0.00065 & 0.00014 \\
\hline 5760.13695 & 2890.0 & I & 0.00039 & -0.00012 & 5759.74364 & 2888.5 & II & 0.00056 & 0.00005 \\
\hline 5760.39929 & 2891.0 & I & 0.00042 & -0.00009 & 5760.00603 & 2889.5 & II & 0.00063 & 0.00012 \\
\hline 5760.66157 & 2892.0 & I & 0.00038 & -0.00013 & 5760.26829 & 2890.5 & II & 0.00058 & 0.00007 \\
\hline 5760.92396 & 2893.0 & I & 0.00046 & -0.00005 & 5760.53054 & 2891.5 & II & 0.00052 & 0.00001 \\
\hline 5761.18628 & 2894.0 & I & 0.00046 & -0.00005 & 5760.79294 & 2892.5 & II & 0.00059 & 0.00008 \\
\hline 5761.44852 & 2895.0 & I & 0.00039 & -0.00012 & 5761.05529 & 2893.5 & II & 0.00063 & 0.00012 \\
\hline 5761.71085 & 2896.0 & I & 0.00040 & -0.00011 & 5761.31752 & 2894.5 & II & 0.00054 & 0.00003 \\
\hline 5761.97323 & 2897.0 & I & 0.00046 & -0.00005 & 5761.57990 & 2895.5 & II & 0.00061 & 0.00010 \\
\hline 5762.23551 & 2898.0 & I & 0.00042 & -0.00009 & 5761.84227 & 2896.5 & II & 0.00066 & 0.00015 \\
\hline 5762.49784 & 2899.0 & I & 0.00043 & -0.00008 & 5762.10455 & 2897.5 & II & 0.00062 & 0.00011 \\
\hline 5762.76015 & 2900.0 & I & 0.00043 & -0.00008 & 5762.36681 & 2898.5 & II & 0.00056 & 0.00005 \\
\hline 5763.02252 & 2901.0 & I & 0.00048 & -0.00003 & 5762.62918 & 2899.5 & II & 0.00062 & 0.00011 \\
\hline 5763.28483 & 2902.0 & I & 0.00047 & -0.00004 & 5762.89124 & 2900.5 & II & 0.00037 & -0.00014 \\
\hline 5763.54712 & 2903.0 & I & 0.00045 & -0.00006 & 5763.15403 & 2901.5 & II & 0.00083 & 0.00032 \\
\hline 5763.80947 & 2904.0 & I & 0.00048 & -0.00003 & 5763.41597 & 2902.5 & II & 0.00046 & -0.00005 \\
\hline 5764.07172 & 2905.0 & I & 0.00041 & -0.00010 & 5763.67842 & 2903.5 & II & 0.00059 & 0.00008 \\
\hline 5764.33411 & 2906.0 & I & 0.00049 & -0.00002 & 5763.94075 & 2904.5 & II & 0.00061 & 0.00010 \\
\hline 5764.59645 & 2907.0 & I & 0.00051 & 0.00000 & 5764.20278 & 2905.5 & II & 0.00032 & -0.00019 \\
\hline 5764.85872 & 2908.0 & I & 0.00047 & -0.00004 & 5764.46537 & 2906.5 & II & 0.00059 & 0.00008 \\
\hline 5765.12109 & 2909.0 & I & 0.00052 & 0.00001 & 5764.72771 & 2907.5 & II & 0.00062 & 0.00011 \\
\hline 5765.38338 & 2910.0 & I & 0.00049 & -0.00002 & 5764.99002 & 2908.5 & II & 0.00061 & 0.00010 \\
\hline 5765.64561 & 2911.0 & I & 0.00040 & -0.00011 & 5765.25234 & 2909.5 & II & 0.00061 & 0.00010 \\
\hline 5765.90796 & 2912.0 & I & 0.00044 & -0.00007 & 5765.51464 & 2910.5 & II & 0.00059 & 0.00008 \\
\hline 5766.17027 & 2913.0 & I & 0.00043 & -0.00008 & 5765.77706 & 2911.5 & II & 0.00070 & 0.00019 \\
\hline 5766.43259 & 2914.0 & I & 0.00043 & -0.00008 & 5766.03932 & 2912.5 & II & 0.00064 & 0.00013 \\
\hline 5766.69492 & 2915.0 & I & 0.00045 & -0.00006 & 5766.30149 & 2913.5 & II & 0.00049 & -0.00002 \\
\hline 5766.95725 & 2916.0 & I & 0.00046 & -0.00005 & 5766.56391 & 2914.5 & II & 0.00059 & 0.00008 \\
\hline 5767.21957 & 2917.0 & I & 0.00046 & -0.00004 & 5766.82620 & 2915.5 & II & 0.00057 & 0.00006 \\
\hline 5767.48203 & 2918.0 & I & 0.00060 & 0.00010 & 5767.08856 & 2916.5 & II & 0.00061 & 0.00010 \\
\hline 5767.74426 & 2919.0 & I & 0.00053 & 0.00002 & 5767.35084 & 2917.5 & II & 0.00057 & 0.00006 \\
\hline
\end{tabular}


TABLE 1 (CONTINUED)

\begin{tabular}{|c|c|c|c|c|c|c|c|c|c|}
\hline $\begin{array}{c}\text { BJD } \\
(+2450000)\end{array}$ & $\mathrm{E}$ & Type & $\begin{array}{c}(O-C)_{I} \\
\quad \text { (day) }\end{array}$ & $\begin{array}{c}(O-C)_{I I} \\
\quad(\text { day })\end{array}$ & $\begin{array}{c}\text { BJD } \\
(+2450000)\end{array}$ & $\mathrm{E}$ & Type & $\begin{array}{c}(O-C)_{I} \\
(\text { day })\end{array}$ & $\begin{array}{c}(O-C)_{I I} \\
\quad \text { (day) }\end{array}$ \\
\hline 5768.00651 & 2920.0 & I & 0.00045 & -0.00006 & 5767.61314 & 2918.5 & II & 0.00056 & 0.00005 \\
\hline 5768.26882 & 2921.0 & I & 0.00044 & -0.00006 & 5767.87550 & 2919.5 & II & 0.00060 & 0.00009 \\
\hline 5768.53113 & 2922.0 & I & 0.00044 & -0.00006 & 5768.13771 & 2920.5 & II & 0.00049 & -0.00001 \\
\hline 5768.79353 & 2923.0 & I & 0.00052 & 0.00002 & 5768.40024 & 2921.5 & II & 0.00071 & 0.00020 \\
\hline 5769.05572 & 2924.0 & I & 0.00040 & -0.00011 & 5768.66241 & 2922.5 & II & 0.00056 & 0.00006 \\
\hline 5769.31808 & 2925.0 & I & 0.00044 & -0.00007 & 5768.92476 & 2923.5 & II & 0.00059 & 0.00009 \\
\hline 5769.58036 & 2926.0 & I & 0.00041 & -0.00010 & 5769.18702 & 2924.5 & II & 0.00053 & 0.00003 \\
\hline 5769.84269 & 2927.0 & I & 0.00042 & -0.00009 & 5769.44938 & 2925.5 & II & 0.00058 & 0.00007 \\
\hline 5770.89201 & 2931.0 & I & 0.00047 & -0.00004 & 5769.71170 & 2926.5 & II & 0.00058 & 0.00007 \\
\hline 5771.15430 & 2932.0 & I & 0.00044 & -0.00007 & 5771.02329 & 2931.5 & II & 0.00059 & 0.00009 \\
\hline 5771.41653 & 2933.0 & I & 0.00035 & -0.00015 & 5771.28561 & 2932.5 & II & 0.00059 & 0.00008 \\
\hline 5771.67891 & 2934.0 & I & 0.00042 & -0.00009 & 5771.54806 & 2933.5 & II & 0.00073 & 0.00022 \\
\hline 5771.94126 & 2935.0 & I & 0.00045 & -0.00006 & 5771.81024 & 2934.5 & II & 0.00059 & 0.00008 \\
\hline 5772.20359 & 2936.0 & I & 0.00047 & -0.00004 & 5772.07252 & 2935.5 & II & 0.00055 & 0.00004 \\
\hline 5772.46592 & 2937.0 & I & 0.00048 & -0.00003 & 5772.33480 & 2936.5 & II & 0.00052 & 0.00001 \\
\hline 5772.72823 & 2938.0 & I & 0.00047 & -0.00003 & 5772.59722 & 2937.5 & II & 0.00062 & 0.00011 \\
\hline 5772.99053 & 2939.0 & I & 0.00046 & -0.00005 & 5772.85951 & 2938.5 & II & 0.00060 & 0.00009 \\
\hline 5773.25281 & 2940.0 & I & 0.00041 & -0.00009 & 5773.12172 & 2939.5 & II & 0.00048 & -0.00002 \\
\hline 5773.77750 & 2942.0 & I & 0.00047 & -0.00004 & 5773.38416 & 2940.5 & II & 0.00061 & 0.00011 \\
\hline 5774.03976 & 2943.0 & I & 0.00042 & -0.00009 & 5773.64643 & 2941.5 & II & 0.00057 & 0.00006 \\
\hline 5774.30213 & 2944.0 & I & 0.00047 & -0.00004 & 5773.90875 & 2942.5 & II & 0.00056 & 0.00005 \\
\hline 5774.56442 & 2945.0 & I & 0.00044 & -0.00007 & 5774.17113 & 2943.5 & II & 0.00063 & 0.00012 \\
\hline 5774.82673 & 2946.0 & I & 0.00044 & -0.00007 & 5774.43336 & 2944.5 & II & 0.00055 & 0.00004 \\
\hline 5775.08902 & 2947.0 & I & 0.00041 & -0.00010 & 5774.69576 & 2945.5 & II & 0.00063 & 0.00012 \\
\hline 5775.35138 & 2948.0 & I & 0.00045 & -0.00006 & 5774.95804 & 2946.5 & II & 0.00059 & 0.00008 \\
\hline 5775.61364 & 2949.0 & I & 0.00040 & -0.00011 & 5775.22027 & 2947.5 & II & 0.00051 & 0.00000 \\
\hline 5775.87595 & 2950.0 & I & 0.00039 & -0.00011 & 5775.48274 & 2948.5 & II & 0.00065 & 0.00014 \\
\hline 5776.13830 & 2951.0 & I & 0.00042 & -0.00009 & 5775.74500 & 2949.5 & II & 0.00060 & 0.00009 \\
\hline 5776.40067 & 2952.0 & I & 0.00047 & -0.00004 & 5776.00734 & 2950.5 & II & 0.00062 & 0.00011 \\
\hline 5776.66298 & 2953.0 & I & 0.00047 & -0.00003 & 5776.26960 & 2951.5 & II & 0.00057 & 0.00006 \\
\hline 5776.92523 & 2954.0 & I & 0.00041 & -0.00010 & 5776.53194 & 2952.5 & II & 0.00059 & 0.00008 \\
\hline 5777.18759 & 2955.0 & I & 0.00044 & -0.00006 & 5776.79429 & 2953.5 & II & 0.00062 & 0.00011 \\
\hline 5777.44985 & 2956.0 & I & 0.00039 & -0.00011 & 5777.05653 & 2954.5 & II & 0.00055 & 0.00004 \\
\hline 5777.71216 & 2957.0 & I & 0.00038 & -0.00013 & 5777.31886 & 2955.5 & II & 0.00056 & 0.00005 \\
\hline 5777.97445 & 2958.0 & I & 0.00036 & -0.00015 & 5777.58118 & 2956.5 & II & 0.00056 & 0.00005 \\
\hline 5778.23677 & 2959.0 & I & 0.00036 & -0.00015 & 5777.84361 & 2957.5 & II & 0.00067 & 0.00017 \\
\hline 5778.49912 & 2960.0 & I & 0.00039 & -0.00011 & 5778.10584 & 2958.5 & II & 0.00059 & 0.00008 \\
\hline 5778.76147 & 2961.0 & I & 0.00042 & -0.00008 & 5778.36816 & 2959.5 & II & 0.00059 & 0.00008 \\
\hline 5779.02378 & 2962.0 & I & 0.00042 & -0.00009 & 5778.63048 & 2960.5 & II & 0.00060 & 0.00009 \\
\hline 5779.28609 & 2963.0 & I & 0.00041 & -0.00009 & 5778.89269 & 2961.5 & II & 0.00049 & -0.00002 \\
\hline 5779.54846 & 2964.0 & I & 0.00047 & -0.00004 & 5779.15504 & 2962.5 & II & 0.00052 & 0.00001 \\
\hline 5779.81070 & 2965.0 & I & 0.00039 & -0.00012 & 5779.41739 & 2963.5 & II & 0.00056 & 0.00005 \\
\hline 5780.07298 & 2966.0 & I & 0.00035 & -0.00015 & 5779.67979 & 2964.5 & II & 0.00063 & 0.00013 \\
\hline 5780.33536 & 2967.0 & I & 0.00042 & -0.00009 & 5779.94215 & 2965.5 & II & 0.00068 & 0.00017 \\
\hline 5780.59768 & 2968.0 & I & 0.00042 & -0.00009 & 5780.20452 & 2966.5 & II & 0.00073 & 0.00022 \\
\hline 5780.85996 & 2969.0 & I & 0.00038 & -0.00012 & 5780.46661 & 2967.5 & II & 0.00051 & 0.00000 \\
\hline 5781.12228 & 2970.0 & I & 0.00039 & -0.00012 & 5780.72892 & 2968.5 & II & 0.00050 & -0.00001 \\
\hline 5781.38458 & 2971.0 & I & 0.00037 & -0.00014 & 5780.99127 & 2969.5 & II & 0.00053 & 0.00003 \\
\hline 5781.64690 & 2972.0 & I & 0.00037 & -0.00014 & 5781.25359 & 2970.5 & II & 0.00053 & 0.00003 \\
\hline 5781.90926 & 2973.0 & I & 0.00042 & -0.00009 & 5781.51601 & 2971.5 & II & 0.00063 & 0.00013 \\
\hline 5782.17159 & 2974.0 & I & 0.00042 & -0.00008 & 5781.77844 & 2972.5 & II & 0.00075 & 0.00025 \\
\hline 5782.43385 & 2975.0 & I & 0.00037 & -0.00014 & 5782.04071 & 2973.5 & II & 0.00071 & 0.00020 \\
\hline 5782.69628 & 2976.0 & I & 0.00049 & -0.00002 & 5782.30292 & 2974.5 & II & 0.00059 & 0.00009 \\
\hline 5782.95845 & 2977.0 & I & 0.00033 & -0.00017 & 5782.56533 & 2975.5 & II & 0.00070 & 0.00019 \\
\hline
\end{tabular}


TABLE 1 (CONTINUED)

\begin{tabular}{|c|c|c|c|c|c|c|c|c|c|}
\hline $\begin{array}{c}\text { BJD } \\
(+2450000)\end{array}$ & $\mathrm{E}$ & Type & $\begin{array}{c}(O-C)_{I} \\
\quad(\text { day })\end{array}$ & $\begin{array}{l}(O-C)_{I I} \\
\quad(\text { day })\end{array}$ & $\begin{array}{c}\text { BJD } \\
(+2450000)\end{array}$ & $\mathrm{E}$ & Type & $\begin{array}{c}(O-C)_{I} \\
\quad(\text { day })\end{array}$ & $\begin{array}{l}(O-C)_{I I} \\
\quad \text { (day) }\end{array}$ \\
\hline 5783.22076 & 2978.0 & I & 0.00033 & -0.00017 & 5782.82763 & 2976.5 & II & 0.00067 & 0.00017 \\
\hline 5783.48316 & 2979.0 & I & 0.00042 & -0.00009 & 5783.08978 & 2977.5 & II & 0.00051 & 0.00000 \\
\hline 5783.74559 & 2980.0 & I & 0.00052 & 0.00002 & 5783.35222 & 2978.5 & II & 0.00063 & 0.00013 \\
\hline 5784.00765 & 2981.0 & I & 0.00027 & -0.00023 & 5783.61454 & 2979.5 & II & 0.00064 & 0.00013 \\
\hline 5784.27018 & 2982.0 & I & 0.00049 & -0.00002 & 5783.87685 & 2980.5 & II & 0.00063 & 0.00012 \\
\hline 5784.53237 & 2983.0 & I & 0.00036 & -0.00015 & 5784.13909 & 2981.5 & II & 0.00055 & 0.00004 \\
\hline 5784.79470 & 2984.0 & I & 0.00037 & -0.00014 & 5784.40146 & 2982.5 & II & 0.00061 & 0.00010 \\
\hline 5785.05708 & 2985.0 & I & 0.00043 & -0.00007 & 5784.66373 & 2983.5 & II & 0.00056 & 0.00005 \\
\hline 5785.31938 & 2986.0 & I & 0.00041 & -0.00009 & 5784.92628 & 2984.5 & II & 0.00079 & 0.00029 \\
\hline 5785.58162 & 2987.0 & I & 0.00033 & -0.00017 & 5785.18850 & 2985.5 & II & 0.00070 & 0.00019 \\
\hline 5785.84400 & 2988.0 & I & 0.00040 & -0.00011 & 5785.45072 & 2986.5 & II & 0.00060 & 0.00010 \\
\hline 5786.10633 & 2989.0 & I & 0.00041 & -0.00009 & 5785.71294 & 2987.5 & II & 0.00050 & 0.00000 \\
\hline 5786.36872 & 2990.0 & I & 0.00049 & -0.00002 & 5786.23768 & 2989.5 & II & 0.00060 & 0.00010 \\
\hline 5786.63076 & 2991.0 & I & 0.00021 & -0.00029 & 5786.49999 & 2990.5 & II & 0.00060 & 0.00009 \\
\hline 5786.89323 & 2992.0 & I & 0.00036 & -0.00014 & 5786.76227 & 2991.5 & II & 0.00057 & 0.00006 \\
\hline 5787.15562 & 2993.0 & I & 0.00044 & -0.00007 & 5787.02466 & 2992.5 & II & 0.00064 & 0.00013 \\
\hline 5787.41789 & 2994.0 & I & 0.00039 & -0.00012 & 5787.28710 & 2993.5 & II & 0.00076 & 0.00025 \\
\hline 5787.68022 & 2995.0 & I & 0.00040 & -0.00011 & 5787.54920 & 2994.5 & II & 0.00054 & 0.00003 \\
\hline 5787.94251 & 2996.0 & I & 0.00038 & -0.00013 & 5787.81157 & 2995.5 & II & 0.00060 & 0.00009 \\
\hline 5788.20475 & 2997.0 & I & 0.00030 & -0.00020 & 5788.07397 & 2996.5 & II & 0.00068 & 0.00018 \\
\hline 5788.46720 & 2998.0 & I & 0.00043 & -0.00007 & 5788.33633 & 2997.5 & II & 0.00072 & 0.00021 \\
\hline 5788.72942 & 2999.0 & I & 0.00034 & -0.00017 & 5788.59842 & 2998.5 & II & 0.00050 & -0.00001 \\
\hline 5788.99177 & 3000.0 & I & 0.00037 & -0.00014 & 5788.86078 & 2999.5 & II & 0.00053 & 0.00003 \\
\hline 5789.25408 & 3001.0 & I & 0.00036 & -0.00014 & 5789.12328 & 3000.5 & II & 0.00072 & 0.00022 \\
\hline 5789.51641 & 3002.0 & I & 0.00038 & -0.00013 & 5789.38556 & 3001.5 & II & 0.00069 & 0.00018 \\
\hline 5789.77866 & 3003.0 & I & 0.00031 & -0.00020 & 5789.64780 & 3002.5 & II & 0.00061 & 0.00010 \\
\hline 5790.04109 & 3004.0 & I & 0.00042 & -0.00008 & 5789.91000 & 3003.5 & II & 0.00050 & -0.00001 \\
\hline 5790.30352 & 3005.0 & I & 0.00053 & 0.00003 & 5790.17263 & 3004.5 & II & 0.00080 & 0.00030 \\
\hline 5790.56574 & 3006.0 & I & 0.00044 & -0.00007 & 5790.43473 & 3005.5 & II & 0.00058 & 0.00008 \\
\hline 5790.82787 & 3007.0 & I & 0.00025 & -0.00025 & 5790.69702 & 3006.5 & II & 0.00056 & 0.00006 \\
\hline 5791.09036 & 3008.0 & I & 0.00042 & -0.00008 & 5790.95941 & 3007.5 & II & 0.00064 & 0.00013 \\
\hline 5791.35262 & 3009.0 & I & 0.00037 & -0.00014 & 5791.22160 & 3008.5 & II & 0.00051 & 0.00000 \\
\hline 5791.61493 & 3010.0 & I & 0.00037 & -0.00014 & 5791.48409 & 3009.5 & II & 0.00068 & 0.00017 \\
\hline 5791.87739 & 3011.0 & I & 0.00050 & 0.00000 & 5791.74635 & 3010.5 & II & 0.00062 & 0.00012 \\
\hline 5792.13963 & 3012.0 & I & 0.00043 & -0.00008 & 5792.00872 & 3011.5 & II & 0.00068 & 0.00017 \\
\hline 5792.40196 & 3013.0 & I & 0.00044 & -0.00007 & 5792.27091 & 3012.5 & II & 0.00055 & 0.00005 \\
\hline 5792.66439 & 3014.0 & I & 0.00056 & 0.00005 & 5792.53334 & 3013.5 & II & 0.00066 & 0.00015 \\
\hline 5792.92667 & 3015.0 & I & 0.00052 & 0.00002 & 5792.79570 & 3014.5 & II & 0.00071 & 0.00020 \\
\hline 5793.18883 & 3016.0 & I & 0.00036 & -0.00014 & 5793.05791 & 3015.5 & II & 0.00059 & 0.00009 \\
\hline 5793.45125 & 3017.0 & I & 0.00046 & -0.00004 & 5793.32014 & 3016.5 & II & 0.00051 & 0.00001 \\
\hline 5793.71356 & 3018.0 & I & 0.00045 & -0.00005 & 5793.58251 & 3017.5 & II & 0.00057 & 0.00007 \\
\hline 5793.97583 & 3019.0 & I & 0.00042 & -0.00009 & 5793.84439 & 3018.5 & II & 0.00013 & -0.00038 \\
\hline 5794.23824 & 3020.0 & I & 0.00050 & 0.00000 & 5794.10726 & 3019.5 & II & 0.00068 & 0.00018 \\
\hline 5794.50047 & 3021.0 & I & 0.00042 & -0.00009 & 5794.36953 & 3020.5 & II & 0.00063 & 0.00013 \\
\hline 5795.02511 & 3023.0 & I & 0.00042 & -0.00008 & 5794.63184 & 3021.5 & II & 0.00063 & 0.00012 \\
\hline 5795.28743 & 3024.0 & I & 0.00042 & -0.00008 & 5794.89418 & 3022.5 & II & 0.00065 & 0.00015 \\
\hline 5795.54970 & 3025.0 & I & 0.00038 & -0.00012 & 5795.15639 & 3023.5 & II & 0.00055 & 0.00004 \\
\hline 5795.81201 & 3026.0 & I & 0.00037 & -0.00013 & 5795.41861 & 3024.5 & II & 0.00045 & -0.00006 \\
\hline 5796.07435 & 3027.0 & I & 0.00040 & -0.00011 & 5795.68117 & 3025.5 & II & 0.00069 & 0.00019 \\
\hline 5796.33660 & 3028.0 & I & 0.00033 & -0.00017 & 5795.94344 & 3026.5 & II & 0.00064 & 0.00014 \\
\hline 5796.59882 & 3029.0 & I & 0.00023 & -0.00027 & 5796.20583 & 3027.5 & II & 0.00072 & 0.00022 \\
\hline 5796.86132 & 3030.0 & I & 0.00042 & -0.00008 & 5796.46806 & 3028.5 & II & 0.00063 & 0.00013 \\
\hline 5797.12378 & 3031.0 & I & 0.00056 & 0.00006 & 5796.73036 & 3029.5 & II & 0.00062 & 0.00011 \\
\hline 5797.38596 & 3032.0 & $\mathrm{I}$ & 0.00042 & -0.00008 & 5796.99260 & 3030.5 & II & 0.00054 & 0.00003 \\
\hline
\end{tabular}


TABLE 1 (CONTINUED)

\begin{tabular}{|c|c|c|c|c|c|c|c|c|c|}
\hline $\begin{array}{c}\text { BJD } \\
(+2450000)\end{array}$ & $\mathrm{E}$ & Type & $\begin{array}{c}(O-C)_{I} \\
\quad \text { (day) }\end{array}$ & $\begin{array}{c}(O-C)_{I I} \\
\quad(\text { day })\end{array}$ & $\begin{array}{c}\text { BJD } \\
(+2450000)\end{array}$ & $\mathrm{E}$ & Type & $\begin{array}{c}(O-C)_{I} \\
\quad \text { (day) }\end{array}$ & $\begin{array}{c}(O-C)_{I I} \\
\quad \text { (day) }\end{array}$ \\
\hline 5797.64831 & 3033.0 & I & 0.00046 & -0.00005 & 5797.25499 & 3031.5 & II & 0.00061 & 0.00011 \\
\hline 5797.91059 & 3034.0 & I & 0.00042 & -0.00008 & 5797.51730 & 3032.5 & II & 0.00060 & 0.00010 \\
\hline 5798.17290 & 3035.0 & I & 0.00041 & -0.00009 & 5797.77958 & 3033.5 & II & 0.00056 & 0.00006 \\
\hline 5798.43522 & 3036.0 & I & 0.00042 & -0.00008 & 5798.04192 & 3034.5 & II & 0.00059 & 0.00009 \\
\hline 5798.69750 & 3037.0 & I & 0.00038 & -0.00012 & 5798.30430 & 3035.5 & II & 0.00065 & 0.00015 \\
\hline 5798.95987 & 3038.0 & I & 0.00044 & -0.00007 & 5798.56658 & 3036.5 & II & 0.00061 & 0.00011 \\
\hline 5799.22219 & 3039.0 & I & 0.00044 & -0.00007 & 5798.82883 & 3037.5 & II & 0.00055 & 0.00004 \\
\hline 5799.48450 & 3040.0 & I & 0.00042 & -0.00008 & 5799.09109 & 3038.5 & II & 0.00049 & -0.00001 \\
\hline 5799.74680 & 3041.0 & I & 0.00041 & -0.00009 & 5799.35343 & 3039.5 & II & 0.00052 & 0.00002 \\
\hline 5800.00915 & 3042.0 & I & 0.00045 & -0.00006 & 5799.61583 & 3040.5 & II & 0.00060 & 0.00010 \\
\hline 5800.27136 & 3043.0 & I & 0.00033 & -0.00017 & 5799.87847 & 3041.5 & II & 0.00092 & 0.00042 \\
\hline 5800.53375 & 3044.0 & I & 0.00041 & -0.00009 & 5800.14042 & 3042.5 & II & 0.00055 & 0.00005 \\
\hline 5800.79601 & 3045.0 & I & 0.00035 & -0.00015 & 5800.40276 & 3043.5 & II & 0.00058 & 0.00007 \\
\hline 5801.05841 & 3046.0 & I & 0.00044 & -0.00006 & 5800.66503 & 3044.5 & II & 0.00053 & 0.00003 \\
\hline 5801.32068 & 3047.0 & I & 0.00039 & -0.00011 & 5800.92739 & 3045.5 & II & 0.00058 & 0.00008 \\
\hline 5801.58306 & 3048.0 & I & 0.00045 & -0.00005 & 5801.18955 & 3046.5 & II & 0.00042 & -0.00008 \\
\hline 5801.84539 & 3049.0 & I & 0.00046 & -0.00004 & 5801.45207 & 3047.5 & II & 0.00062 & 0.00012 \\
\hline 5803.15703 & 3054.0 & I & 0.00052 & 0.00002 & 5801.71447 & 3048.5 & II & 0.00070 & 0.00020 \\
\hline 5803.41922 & 3055.0 & I & 0.00040 & -0.00010 & 5803.28830 & 3054.5 & II & 0.00064 & 0.00013 \\
\hline 5803.68156 & 3056.0 & I & 0.00042 & -0.00008 & 5803.55064 & 3055.5 & II & 0.00065 & 0.00015 \\
\hline 5803.94397 & 3057.0 & I & 0.00051 & 0.00001 & 5803.81290 & 3056.5 & II & 0.00061 & 0.00010 \\
\hline 5804.20622 & 3058.0 & I & 0.00044 & -0.00006 & 5804.07518 & 3057.5 & II & 0.00056 & 0.00006 \\
\hline 5804.46852 & 3059.0 & I & 0.00043 & -0.00007 & 5804.33752 & 3058.5 & II & 0.00059 & 0.00009 \\
\hline 5804.73098 & 3060.0 & I & 0.00057 & 0.00007 & 5804.59987 & 3059.5 & II & 0.00062 & 0.00012 \\
\hline 5804.99308 & 3061.0 & I & 0.00036 & -0.00015 & 5804.86217 & 3060.5 & II & 0.00060 & 0.00010 \\
\hline 5805.25550 & 3062.0 & I & 0.00046 & -0.00004 & 5805.12447 & 3061.5 & II & 0.00059 & 0.00008 \\
\hline 5805.51784 & 3063.0 & I & 0.00048 & -0.00002 & 5805.38688 & 3062.5 & II & 0.00068 & 0.00018 \\
\hline 5805.78006 & 3064.0 & I & 0.00038 & -0.00012 & 5805.64934 & 3063.5 & II & 0.00082 & 0.00032 \\
\hline 5806.04238 & 3065.0 & I & 0.00039 & -0.00011 & 5805.91151 & 3064.5 & II & 0.00068 & 0.00018 \\
\hline 5806.30477 & 3066.0 & I & 0.00046 & -0.00005 & 5806.17373 & 3065.5 & II & 0.00058 & 0.00008 \\
\hline 5806.56712 & 3067.0 & I & 0.00050 & -0.00001 & 5806.43608 & 3066.5 & II & 0.00062 & 0.00011 \\
\hline 5806.82940 & 3068.0 & I & 0.00045 & -0.00005 & 5806.69838 & 3067.5 & II & 0.00059 & 0.00009 \\
\hline 5807.09167 & 3069.0 & I & 0.00041 & -0.00009 & 5806.96067 & 3068.5 & II & 0.00057 & 0.00007 \\
\hline 5807.35401 & 3070.0 & I & 0.00043 & -0.00007 & 5807.22302 & 3069.5 & II & 0.00060 & 0.00010 \\
\hline 5807.61627 & 3071.0 & I & 0.00038 & -0.00012 & 5807.74772 & 3071.5 & II & 0.00067 & 0.00017 \\
\hline 5807.87864 & 3072.0 & I & 0.00043 & -0.00008 & 5808.00991 & 3072.5 & II & 0.00054 & 0.00004 \\
\hline 5808.14096 & 3073.0 & I & 0.00043 & -0.00007 & 5808.27221 & 3073.5 & II & 0.00053 & 0.00002 \\
\hline 5808.40327 & 3074.0 & I & 0.00043 & -0.00007 & 5808.53459 & 3074.5 & II & 0.00059 & 0.00009 \\
\hline 5808.66558 & 3075.0 & I & 0.00042 & -0.00008 & 5808.79695 & 3075.5 & II & 0.00063 & 0.00013 \\
\hline 5808.92788 & 3076.0 & I & 0.00040 & -0.00010 & 5809.05922 & 3076.5 & II & 0.00059 & 0.00008 \\
\hline 5809.19021 & 3077.0 & I & 0.00042 & -0.00008 & 5809.32162 & 3077.5 & II & 0.00067 & 0.00017 \\
\hline 5809.71445 & 3079.0 & I & 0.00003 & -0.00047 & 5809.58393 & 3078.5 & II & 0.00066 & 0.00016 \\
\hline 5809.97705 & 3080.0 & I & 0.00030 & -0.00020 & 5809.84621 & 3079.5 & II & 0.00063 & 0.00013 \\
\hline 5810.23950 & 3081.0 & I & 0.00044 & -0.00006 & 5810.10852 & 3080.5 & II & 0.00062 & 0.00012 \\
\hline 5810.50178 & 3082.0 & I & 0.00040 & -0.00010 & 5810.37081 & 3081.5 & II & 0.00059 & 0.00009 \\
\hline 5810.76405 & 3083.0 & I & 0.00035 & -0.00015 & 5810.63311 & 3082.5 & II & 0.00057 & 0.00007 \\
\hline 5811.02640 & 3084.0 & I & 0.00039 & -0.00011 & 5810.89550 & 3083.5 & II & 0.00064 & 0.00014 \\
\hline 5811.28878 & 3085.0 & I & 0.00045 & -0.00005 & 5811.15768 & 3084.5 & II & 0.00051 & 0.00001 \\
\hline 5811.55104 & 3086.0 & I & 0.00040 & -0.00010 & 5811.42012 & 3085.5 & II & 0.00063 & 0.00013 \\
\hline 5811.81335 & 3087.0 & I & 0.00039 & -0.00011 & 5811.68243 & 3086.5 & II & 0.00063 & 0.00013 \\
\hline 5812.07572 & 3088.0 & I & 0.00044 & -0.00006 & 5811.94482 & 3087.5 & II & 0.00070 & 0.00020 \\
\hline 5812.33787 & 3089.0 & I & 0.00028 & -0.00022 & 5812.20700 & 3088.5 & II & 0.00057 & 0.00007 \\
\hline 5812.60016 & 3090.0 & I & 0.00025 & -0.00025 & 5812.46930 & 3089.5 & II & 0.00055 & 0.00005 \\
\hline 5812.86218 & 3091.0 & I & -0.00004 & -0.00055 & 5812.73176 & 3090.5 & II & 0.00069 & 0.00019 \\
\hline
\end{tabular}


TABLE 1 (CONTINUED)

\begin{tabular}{|c|c|c|c|c|c|c|c|c|c|}
\hline $\begin{array}{c}\text { BJD } \\
(+2450000)\end{array}$ & $\mathrm{E}$ & Type & $\begin{array}{c}(O-C)_{I} \\
\quad(\text { day })\end{array}$ & $\begin{array}{l}(O-C)_{I I} \\
\quad(\text { day })\end{array}$ & $\begin{array}{c}\text { BJD } \\
(+2450000)\end{array}$ & $\mathrm{E}$ & Type & $\begin{array}{c}(O-C)_{I} \\
\quad(\text { day })\end{array}$ & $\begin{array}{l}(O-C)_{I I} \\
\quad \text { (day) }\end{array}$ \\
\hline 5813.12485 & 3092.0 & I & 0.00030 & -0.00020 & 5812.99405 & 3091.5 & II & 0.00066 & 0.00016 \\
\hline 5813.38697 & 3093.0 & I & 0.00010 & -0.00040 & 5813.51857 & 3093.5 & II & 0.00055 & 0.00005 \\
\hline 5813.64950 & 3094.0 & I & 0.00032 & -0.00018 & 5813.78083 & 3094.5 & II & 0.00049 & -0.00001 \\
\hline 5813.91198 & 3095.0 & I & 0.00048 & -0.00002 & 5814.04320 & 3095.5 & II & 0.00055 & 0.00005 \\
\hline 5814.17418 & 3096.0 & I & 0.00037 & -0.00013 & 5814.30553 & 3096.5 & II & 0.00056 & 0.00006 \\
\hline 5814.43651 & 3097.0 & I & 0.00038 & -0.00013 & 5814.56796 & 3097.5 & II & 0.00067 & 0.00017 \\
\hline 5814.69876 & 3098.0 & I & 0.00031 & -0.00019 & 5814.83014 & 3098.5 & II & 0.00054 & 0.00004 \\
\hline 5814.96114 & 3099.0 & I & 0.00037 & -0.00013 & 5815.09257 & 3099.5 & II & 0.00065 & 0.00015 \\
\hline 5815.22344 & 3100.0 & I & 0.00036 & -0.00014 & 5815.35425 & 3100.5 & II & 0.00001 & -0.00049 \\
\hline 5815.48564 & 3101.0 & I & 0.00024 & -0.00026 & 5815.61713 & 3101.5 & II & 0.00057 & 0.00007 \\
\hline 5815.74811 & 3102.0 & I & 0.00040 & -0.00010 & 5815.87944 & 3102.5 & II & 0.00057 & 0.00007 \\
\hline 5816.01052 & 3103.0 & I & 0.00049 & -0.00001 & 5816.14174 & 3103.5 & II & 0.00055 & 0.00005 \\
\hline 5816.27266 & 3104.0 & I & 0.00031 & -0.00019 & 5816.40395 & 3104.5 & II & 0.00044 & -0.00006 \\
\hline 5816.53504 & 3105.0 & I & 0.00037 & -0.00013 & 5816.66593 & 3105.5 & II & 0.00010 & -0.00040 \\
\hline 5816.79728 & 3106.0 & I & 0.00030 & -0.00020 & 5816.92880 & 3106.5 & II & 0.00066 & 0.00016 \\
\hline 5817.05964 & 3107.0 & I & 0.00034 & -0.00016 & 5817.19100 & 3107.5 & II & 0.00054 & 0.00004 \\
\hline 5817.32198 & 3108.0 & I & 0.00037 & -0.00013 & 5817.45352 & 3108.5 & II & 0.00075 & 0.00025 \\
\hline 5817.58428 & 3109.0 & I & 0.00035 & -0.00015 & 5817.71574 & 3109.5 & II & 0.00065 & 0.00015 \\
\hline 5817.84666 & 3110.0 & I & 0.00041 & -0.00009 & 5817.97813 & 3110.5 & II & 0.00073 & 0.00023 \\
\hline 5818.10956 & 3111.0 & I & 0.00100 & 0.00050 & 5818.24076 & 3111.5 & II & 0.00104 & 0.00054 \\
\hline 5818.37127 & 3112.0 & I & 0.00039 & -0.00011 & 5818.50250 & 3112.5 & II & 0.00046 & -0.00004 \\
\hline 5818.63346 & 3113.0 & I & 0.00026 & -0.00024 & 5818.76494 & 3113.5 & II & 0.00058 & 0.00008 \\
\hline 5818.89586 & 3114.0 & I & 0.00034 & -0.00016 & 5819.02721 & 3114.5 & II & 0.00054 & 0.00004 \\
\hline 5819.15825 & 3115.0 & I & 0.00041 & -0.00009 & 5819.28957 & 3115.5 & II & 0.00058 & 0.00008 \\
\hline 5819.42062 & 3116.0 & I & 0.00047 & -0.00003 & 5819.55194 & 3116.5 & II & 0.00063 & 0.00013 \\
\hline 5819.68287 & 3117.0 & I & 0.00040 & -0.00010 & 5819.81412 & 3117.5 & II & 0.00050 & 0.00000 \\
\hline 5819.94514 & 3118.0 & I & 0.00036 & -0.00014 & 5820.07665 & 3118.5 & II & 0.00071 & 0.00021 \\
\hline 5820.20747 & 3119.0 & I & 0.00037 & -0.00013 & 5820.33886 & 3119.5 & II & 0.00061 & 0.00011 \\
\hline 5820.46980 & 3120.0 & I & 0.00038 & -0.00012 & 5820.60122 & 3120.5 & II & 0.00064 & 0.00014 \\
\hline 5820.73204 & 3121.0 & I & 0.00031 & -0.00019 & 5820.86365 & 3121.5 & II & 0.00076 & 0.00026 \\
\hline 5820.99433 & 3122.0 & I & 0.00028 & -0.00022 & 5821.12575 & 3122.5 & II & 0.00054 & 0.00004 \\
\hline 5821.25663 & 3123.0 & I & 0.00026 & -0.00024 & 5821.38816 & 3123.5 & II & 0.00064 & 0.00014 \\
\hline 5821.51917 & 3124.0 & I & 0.00049 & -0.00001 & 5821.65047 & 3124.5 & II & 0.00063 & 0.00013 \\
\hline 5821.78135 & 3125.0 & I & 0.00035 & -0.00015 & 5821.91268 & 3125.5 & II & 0.00052 & 0.00002 \\
\hline 5822.04365 & 3126.0 & I & 0.00033 & -0.00017 & 5822.17504 & 3126.5 & II & 0.00057 & 0.00007 \\
\hline 5822.30599 & 3127.0 & I & 0.00036 & -0.00014 & 5822.43737 & 3127.5 & II & 0.00058 & 0.00008 \\
\hline 5822.56831 & 3128.0 & I & 0.00036 & -0.00014 & 5822.69969 & 3128.5 & II & 0.00058 & 0.00008 \\
\hline 5822.83056 & 3129.0 & I & 0.00029 & -0.00021 & 5822.96218 & 3129.5 & II & 0.00075 & 0.00025 \\
\hline 5823.09302 & 3130.0 & I & 0.00044 & -0.00006 & 5823.22431 & 3130.5 & II & 0.00057 & 0.00007 \\
\hline 5823.35536 & 3131.0 & I & 0.00046 & -0.00004 & 5823.48652 & 3131.5 & II & 0.00046 & -0.00004 \\
\hline 5823.61756 & 3132.0 & I & 0.00034 & -0.00016 & 5823.74905 & 3132.5 & II & 0.00067 & 0.00018 \\
\hline 5823.88003 & 3133.0 & I & 0.00049 & -0.00001 & 5824.01127 & 3133.5 & II & 0.00058 & 0.00008 \\
\hline 5824.14225 & 3134.0 & I & 0.00040 & -0.00010 & 5824.27361 & 3134.5 & II & 0.00060 & 0.00010 \\
\hline 5824.40453 & 3135.0 & I & 0.00036 & -0.00014 & 5824.53601 & 3135.5 & II & 0.00068 & 0.00018 \\
\hline 5824.66695 & 3136.0 & I & 0.00047 & -0.00003 & 5824.79824 & 3136.5 & II & 0.00060 & 0.00010 \\
\hline 5824.92924 & 3137.0 & I & 0.00044 & -0.00006 & 5825.06050 & 3137.5 & II & 0.00054 & 0.00004 \\
\hline 5825.19154 & 3138.0 & I & 0.00043 & -0.00007 & 5825.32279 & 3138.5 & II & 0.00051 & 0.00001 \\
\hline 5825.45390 & 3139.0 & I & 0.00046 & -0.00003 & 5825.58529 & 3139.5 & II & 0.00070 & 0.00020 \\
\hline 5825.71621 & 3140.0 & I & 0.00046 & -0.00004 & 5825.84753 & 3140.5 & II & 0.00062 & 0.00012 \\
\hline 5825.97848 & 3141.0 & I & 0.00041 & -0.00009 & 5826.10994 & 3141.5 & II & 0.00071 & 0.00021 \\
\hline 5826.24082 & 3142.0 & I & 0.00043 & -0.00006 & 5826.37214 & 3142.5 & II & 0.00060 & 0.00010 \\
\hline 5826.50316 & 3143.0 & I & 0.00045 & -0.00005 & 5826.63450 & 3143.5 & II & 0.00063 & 0.00014 \\
\hline 5826.76539 & 3144.0 & I & 0.00037 & -0.00013 & 5826.89681 & 3144.5 & II & 0.00063 & 0.00013 \\
\hline 5827.02783 & 3145.0 & $\mathrm{I}$ & 0.00049 & -0.00001 & 5827.15911 & 3145.5 & II & 0.00062 & 0.00012 \\
\hline
\end{tabular}


TABLE 1 (CONTINUED)

\begin{tabular}{|c|c|c|c|c|c|c|c|c|c|}
\hline $\begin{array}{c}\text { BJD } \\
(+2450000)\end{array}$ & $\mathrm{E}$ & Type & $\begin{array}{c}(O-C)_{I} \\
\quad(\text { day })\end{array}$ & $\begin{array}{l}(O-C)_{I I} \\
\quad(\text { day })\end{array}$ & $\begin{array}{c}\text { BJD } \\
(+2450000)\end{array}$ & $\mathrm{E}$ & Type & $\begin{array}{c}(O-C)_{I} \\
\quad(\text { day })\end{array}$ & $\begin{array}{c}(O-C)_{I I} \\
\quad(\text { day })\end{array}$ \\
\hline 5827.29006 & 3146.0 & I & 0.00041 & -0.00009 & 5827.42147 & 3146.5 & II & 0.00066 & 0.00016 \\
\hline 5827.55248 & 3147.0 & I & 0.00051 & 0.00001 & 5827.68367 & 3147.5 & II & 0.00054 & 0.00005 \\
\hline 5827.81476 & 3148.0 & I & 0.00048 & -0.00002 & 5827.94613 & 3148.5 & II & 0.00069 & 0.00019 \\
\hline 5828.07705 & 3149.0 & I & 0.00044 & -0.00005 & 5828.20842 & 3149.5 & II & 0.00066 & 0.00016 \\
\hline 5828.33942 & 3150.0 & I & 0.00050 & 0.00000 & 5828.47068 & 3150.5 & II & 0.00060 & 0.00010 \\
\hline 5828.60162 & 3151.0 & I & 0.00038 & -0.00012 & 5828.73306 & 3151.5 & II & 0.00066 & 0.00016 \\
\hline 5828.86430 & 3152.0 & I & 0.00074 & 0.00024 & 5828.99522 & 3152.5 & II & 0.00051 & 0.00001 \\
\hline 5829.12629 & 3153.0 & I & 0.00042 & -0.00008 & 5829.25755 & 3153.5 & II & 0.00052 & 0.00003 \\
\hline 5829.38866 & 3154.0 & I & 0.00047 & -0.00003 & 5829.51981 & 3154.5 & II & 0.00046 & -0.00004 \\
\hline 5829.65093 & 3155.0 & I & 0.00043 & -0.00007 & 5829.78236 & 3155.5 & II & 0.00069 & 0.00020 \\
\hline 5829.91329 & 3156.0 & I & 0.00047 & -0.00003 & 5830.04457 & 3156.5 & II & 0.00059 & 0.00009 \\
\hline 5830.17562 & 3157.0 & I & 0.00048 & -0.00002 & 5830.30680 & 3157.5 & II & 0.00051 & 0.00001 \\
\hline 5830.43787 & 3158.0 & I & 0.00042 & -0.00008 & 5830.56913 & 3158.5 & II & 0.00051 & 0.00002 \\
\hline 5830.70019 & 3159.0 & I & 0.00042 & -0.00008 & 5830.83157 & 3159.5 & II & 0.00064 & 0.00014 \\
\hline 5830.96248 & 3160.0 & I & 0.00039 & -0.00011 & 5831.09376 & 3160.5 & II & 0.00052 & 0.00002 \\
\hline 5831.22489 & 3161.0 & I & 0.00049 & -0.00001 & 5831.35620 & 3161.5 & II & 0.00064 & 0.00014 \\
\hline 5831.48711 & 3162.0 & I & 0.00039 & -0.00011 & 5831.61846 & 3162.5 & II & 0.00058 & 0.00008 \\
\hline 5831.74944 & 3163.0 & I & 0.00040 & -0.00010 & 5831.88079 & 3163.5 & II & 0.00059 & 0.00009 \\
\hline 5832.01179 & 3164.0 & I & 0.00044 & -0.00006 & 5832.14315 & 3164.5 & II & 0.00064 & 0.00014 \\
\hline 5832.27407 & 3165.0 & I & 0.00039 & -0.00010 & 5832.40538 & 3165.5 & II & 0.00055 & 0.00005 \\
\hline 5832.53641 & 3166.0 & I & 0.00042 & -0.00007 & 5832.66774 & 3166.5 & II & 0.00059 & 0.00010 \\
\hline 5832.79871 & 3167.0 & $\mathrm{I}$ & 0.00041 & -0.00009 & 5832.92998 & 3167.5 & II & 0.00052 & 0.00002 \\
\hline
\end{tabular}

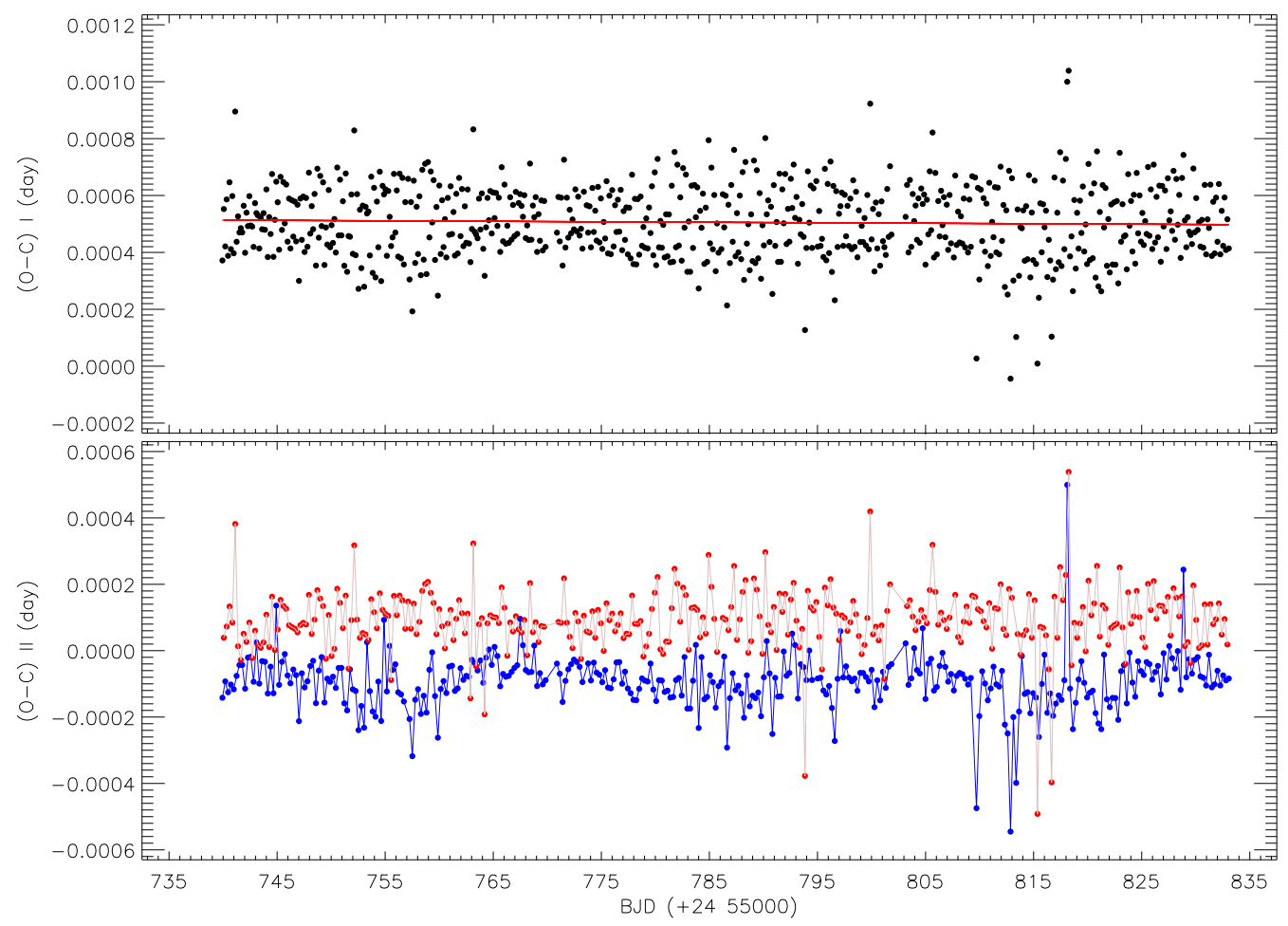

Fig. 2. The variations of time of minimum residuals of $(O-C)_{I}$ and $(O-C)_{I I}$ obtained by applying a linear correction. In the bottom panel, the filled blue circles represent the primary minima; the filled red circles represent the secondary minima. In the upper panel all the residuals are plotted with filled black circles, while the red line represents a linear fit. The color figure can be viewed online. 


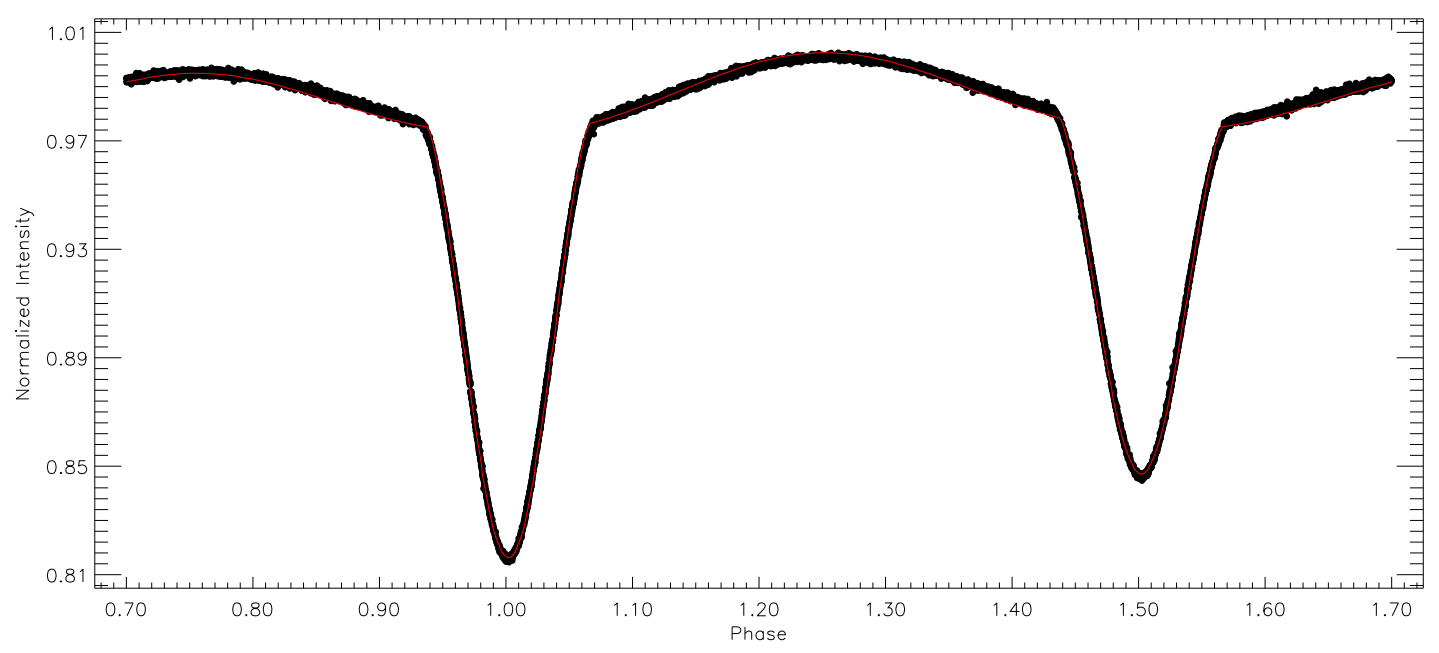

Fig. 3. The light curve along the orbital cycle, from BJD 2455739.94 to 2455829.13. In the figure, the filled circles represent the observations, while the red smooth line represents the synthetic light curve. The color figure can be viewed online.

were tried in the light curve analyses, astrophysically acceptable parameters were obtained only for the detached system mode.

The PHOEBE V.0.32, needs a temperature value for the primary component. We determined its temperature from the $J H K$ brightness given by Cutri et al. (2003). The de-reddened colors were found to be $(H-K)_{\circ}=0^{\mathrm{m}} .252$ and $(J-K)_{\circ}=0^{\mathrm{m}} .629$. Then a temperature value of $4220 \pm 20 \mathrm{~K}$ corresponding to the de-reddened colours was obtained by using the calibrations given by Tokunaga (2000). We accepted this value as the primary component temperature. The temperature of the secondary component was taken as an adjustable free parameter. In the analyses, some coefficients, such as the albedos $\left(A_{1}\right.$ and $\left.A_{2}\right)$, the gravity-darkening coefficients $\left(g_{1}\right.$ and $\left.g_{2}\right)$ and the limb-darkening coefficients $\left(x_{1}\right.$ and $\left.x_{2}\right)$, were taken from the tables given by Lucy (1967); Rucinski (1969); Van Hamme (1993), considering the possible temperatures of both components. The rest of the parameters, such as the dimensionless potentials $\left(\Omega_{1}\right.$ and $\left.\Omega_{2}\right)$, the fractional luminosity $\left(L_{1}\right)$ of the primary component, the inclination $(i)$ and the mass ratio $(q)$ of the system, were taken as adjustable free parameters.

The computed values of the free parameters are tabulated in Table 2, while the synthetic light curve derived by these parameters is shown in Figure 3. As the table shows, some errors are smaller than the expected values. However, the errors given in the table were computed by the PHOEBE V.0.32, depending on Taylor (1997). In fact the $\chi^{2}$ was computed as $3.15 \times 10^{-3}$ from the Kepler short cadence data. The
3D model of Roche geometry obtained with these parameters is shown in Figure 4.

It should be noted that we obtained a solution after some iterations in the detached system mode of the Wilson-Devinney Code (Wilson \& Devinney 1971; Wilson 1990). Although the synthetic curve fitted the observations very well, it was revealed that it did not fit the observations around phase 0.27 . To solve this mismatched part of synthetic light curve, we assumed that there is a spotted area on a component, considering the flare activity. We assumed that the spotted area was on the primary component. It could be assumed that the spotted star was the secondary component, which would lead to another acceptable solution.

The $(B-V)$ color indexes were computed as $1^{\mathrm{m}} .233$ and $1^{\mathrm{m}} .329$ for the primary and secondary components, respectively. The computed color indexes are in agreement with the values found by Walkowicz \& Basri (2013). Considering these values, we determined the masses as $0.644 M_{\odot}$ and $0.570 M_{\odot}$ for primary and secondary components. Then, the semi-major axis of the system was found to be $1.84 R_{\odot}(0.0086 \mathrm{AU})$ according to Kepler's third law. With this value for the semi-major axis, the radius of the primary component was found to be $0.701 R_{\odot}$, while that of the secondary was $0.650 R_{\odot}$.

\subsection{Flare Activity and the OPEA Model}

The main subject of this paper is about flare activity in KIC 12004834. To demonstrate the nature of the flare activity occurring in a star with 
TABLE 2

PARAMETERS OBTAINED FROM THE LIGHT CURVE ANALYSIS OF KIC 12004834

\begin{tabular}{lc}
\hline \multicolumn{1}{c}{ Parameter } & Value \\
\hline$q$ & $0.743 \pm 0.001$ \\
$i\left({ }^{\circ}\right)$ & $75.89 \pm 0.03$ \\
$T_{1}(\mathrm{~K})$ & 4220 (fixed) \\
$T_{2}(\mathrm{~K})$ & $4001 \pm 11$ \\
$\Omega_{1}$ & $4.308 \pm 0.004$ \\
$\Omega_{2}$ & $5.485 \pm 0.007$ \\
$L_{1} / L_{2}$ & $0.245 \pm 0.067$ \\
$g_{1}, g_{2}$ & 0.32 (fixed) \\
$A_{1}, A_{2}$ & 0.32 (fixed) \\
$x_{1}$, bol,$x_{2}$, bol & $0.377,0.001$ (fixed) \\
$x_{1}, x_{2}$ & $0.369,0.001$ (fixed) \\
$<r_{1}>$ & $0.287 \pm 0.001$ \\
$<r_{2}>$ & $0.172 \pm 0.001$ \\
Co - Lat $_{\text {Spot }}^{(\text {rad })}$ & $1.920 \pm 0.004$ \\
Long $_{\text {Spot }}^{(\text {rad }}$ & $1.710 \pm 0.002$ \\
$R_{\text {Spot }}^{(\text {rad }}$ & $0.244 \pm 0.003$ \\
$T_{\text {fSpot }}$ & $0.960 \pm 0.001$ \\
\hline
\end{tabular}

known photometric data, Dal \& Evren (2010) and Dal (2012) described a simple way depending on the method mentioned by Gershberg (1972), which is just a smoothing out of the flares. However, apart from the flare activity, the light curve of KIC 12004834 exhibits other effects caused by both the geometrical nature of the components and the structures on them, such as eclipses and cool spots.

In order to determine and analyze the flares, first we removed the variations seen out-of-flares in the light curves. We follow the method of Dal \& Evren (2010) for KIC 12004834. For this purpose, using the synthetic light curve derived from the light curve analyses described in the previous section, the residual data were obtained as a pre-whitened light curve, in which there was only flare activity variation. Following Dal (2012), 149 flares were detected from the available short cadence data. In the analyses, the synthetic light curve allowed us to fix the quiescent levels at the flare moment, which are shown in Figure 5 .

Taking into account that the luminosity parameter enters in the energy calculations as described by Dal \& Evren (2010, 2011), the equivalent duration parameter was computed for each flare, instead of its energy. According to the beginning and the end of a
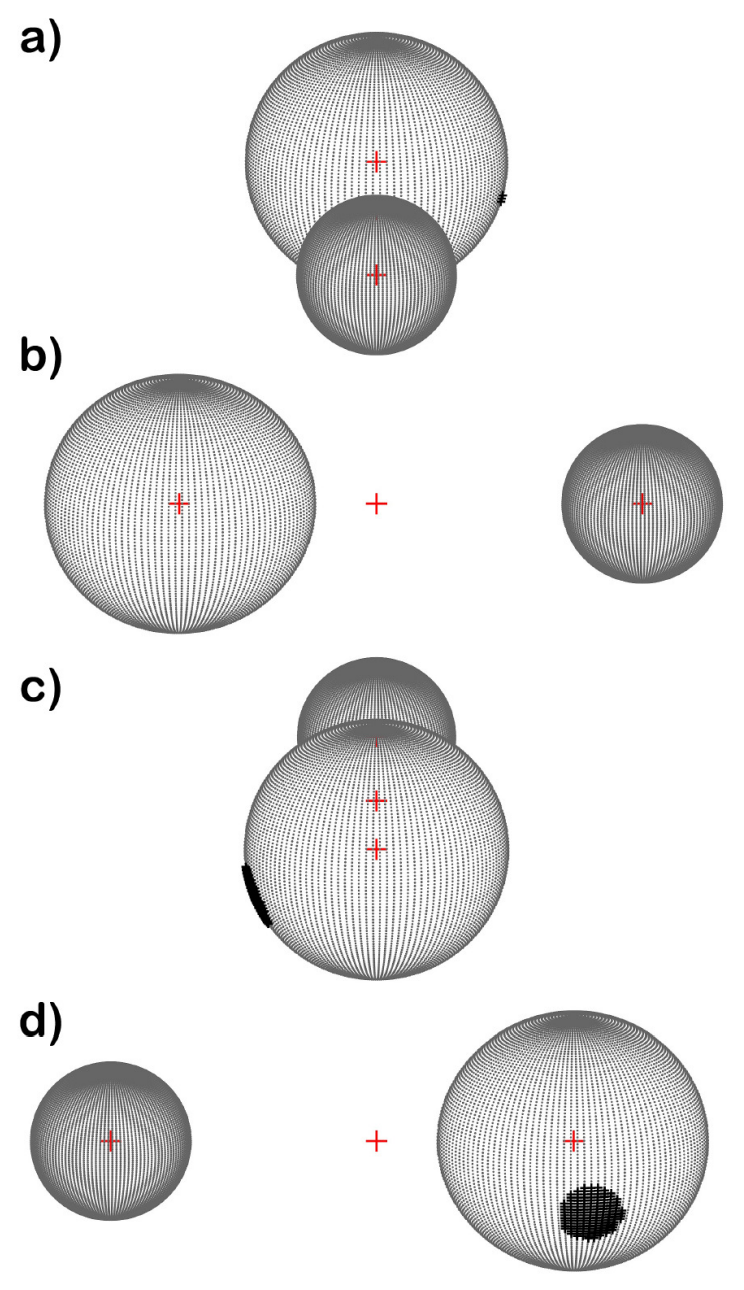

Fig. 4. The 3D model of Roche geometry and spotted area distribution derived from the light curve analysis is shown for different phases, such as (a) 0.00, (b) 0.25, (c) 0.50, (d) 0.75 . The color figure can be viewed online.

flare, the desired parameters, such as flare rise times $\left(T_{r}\right)$, decay times $\left(T_{d}\right)$, amplitudes of flare maxima, flare equivalent durations $(P)$, were computed for each flare. The flare equivalent durations were computed by equation (2) taken from Gershberg (1972):

$$
P=\int\left[\left(I_{\text {flare }}-I_{0}\right) / I_{0}\right] d t
$$

where $I_{0}$ is the flux of the star in the quiet state. As described above, the synthetic light curve derived by the light curve analysis was taken as $I_{0} . I_{\text {flare }}$ is the intensity observed at the moment of the flare. $P$ is the flare-equivalent duration in the observing band. All the computed parameters are listed in Table 3 for these 149 flares. The general standard errors were 

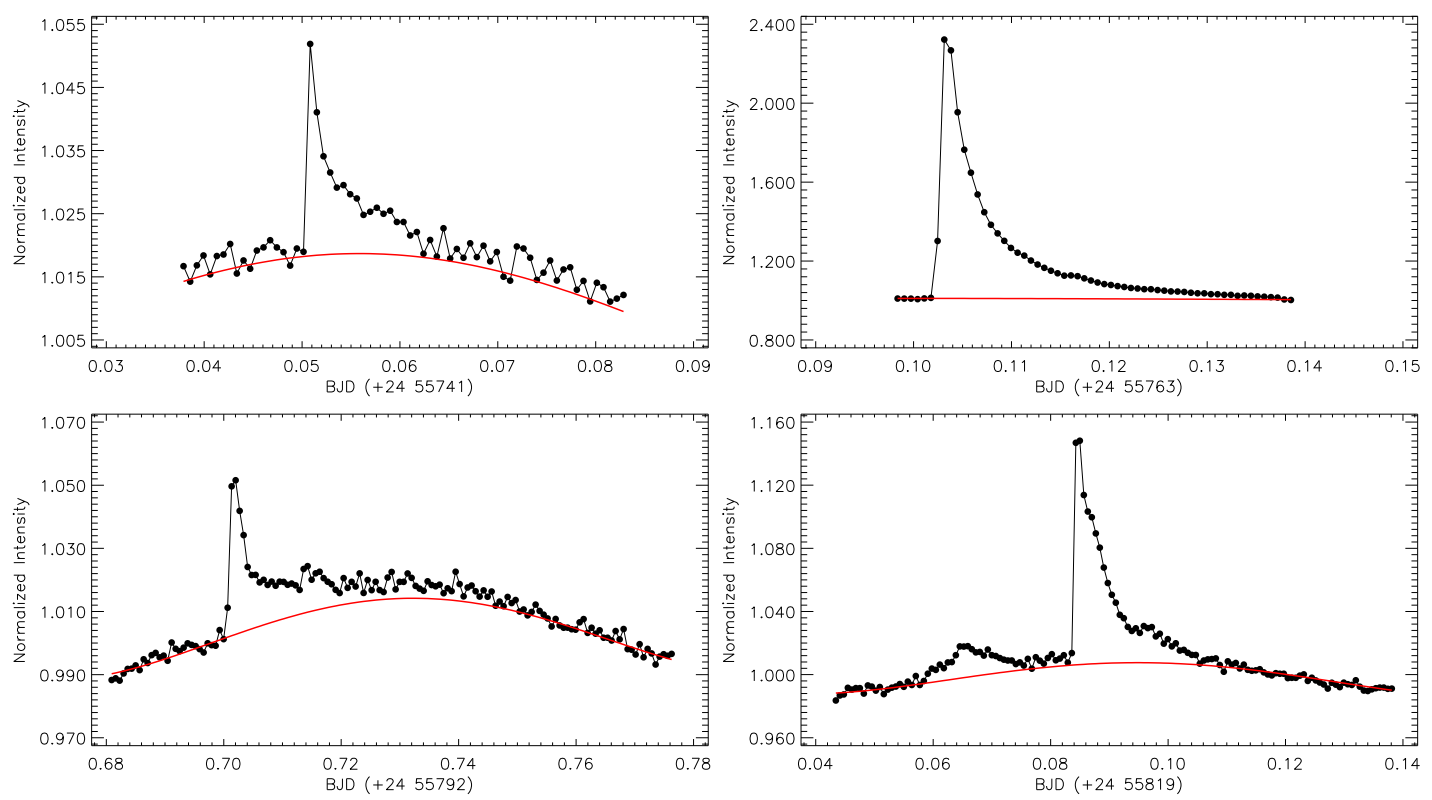

Fig. 5. Four different samples for the flare light variations are shown. In the figure, the filled circles represent the observations, while the (red) lines represent the synthetic light curve obtained from the light curve analysis, which was taken as the quiescent levels for each flare. The color figure can be viewed online.

computed by using the methods described by Taylor (1997) for the time scales such as flare rise and decay times.

The distributions of flare equivalent durations on a logarithmic scale versus flare total durations were modelled by the One Phase Exponential Association (hereafter OPEA) defined by equation (3), using the SPSS V17.0 (Green et al. 1999) and GrahpPad Prism V5.02 (Dawson \& Trapp 2004) software:

$$
y=y_{0}+\left(\text { Plateau }-y_{0}\right) \times\left(1-e^{-k \times x}\right)
$$

where $y$ is the flare equivalent duration, $x$ is the flare total duration. According to the description of Dal \& Evren (2010), the most important parameter in this equation is the Plateau term to reveal the flare behavior of a star. Following three different methods described by D'Agostino \& Stephens (1986), the probability values $(p-$ value $)$ were calculated to test the quality of the fit. The $p-$ value was found to be $<0.001$ in all the methods. The derived OPEA model is shown in Figure 6 together with the observed flare equivalent durations. The parameters computed from the model are listed in Table 4.

In contrast to the known UV Ceti flare stars, KIC 12004834 is a binary system. It is well known that the flare events are generally random phenomena. To test this situation for a close binary like KIC 12004834, we calculated the phase distribution of the flares, depending on the orbital period of the system. The phase distribution is shown in Figure 7. In the figure, the distribution of the total number of flares computed in phase intervals of 0.05 , for all 149 flares is shown.

We also derived the flare energy spectrum (Gershberg 1972) for KIC 12004834. Like for the OPEA model, we again used the flare equivalent duration instead of the flare energy. To derive the flare energy spectrum, the cumulative flare frequencies were computed for 149 flares, and then, its distribution was derived in order to compare KIC 12004834 to its analogues. The obtained cumulative flare frequency distribution and its models are shown in Figure 8.

In the literature there are two other flare frequency descriptions. Contrary to Gershberg (1972), Ishida et al. (1991) described the flare frequencies $N_{1}$ and $N_{2}$, a flare number and a total flare-equivalent duration emitting per hour, respectively. In this study, we detected 149 flares from the available observations lasting 89.19 days. We computed the frequencies by equations (4) and (5) (Ishida et al. 1991):

$$
\begin{aligned}
& N_{1}=\Sigma n_{f} / \Sigma D T \\
& N_{2}=\Sigma P / \Sigma D T
\end{aligned}
$$

where $\Sigma n_{f}$ is the total flare number, $\Sigma D T$ is the total observing duration, and $\Sigma P$ is the total equivalent duration. We found that the $N_{1}$ frequency is $0.070 \mathrm{~h}^{-1}$, while the $N_{2}$ frequency is $0.62 \mathrm{sec}-$ ond/hour for KIC 12004834. It should be noted that 
TABLE 3

THE FLARE PARAMETERS COMPUTED FROM KIC 12004834'S THE AVAILABLE SHORT CADENCE DATA IN THE KEPLER MISSION DATABASE

\begin{tabular}{|c|c|c|c|c|c|}
\hline $\begin{array}{c}\text { Tmax } \\
(B J D-2450000)\end{array}$ & $\begin{array}{l}P \\
(\mathrm{~s})\end{array}$ & $\begin{array}{l}T_{r} \\
(\mathrm{~s})\end{array}$ & $\begin{array}{l}T_{d} \\
(\mathrm{~s})\end{array}$ & $\begin{array}{l}T_{t} \\
(\mathrm{~s})\end{array}$ & $\begin{array}{c}\text { Amplitude } \\
\text { (Relative Flux) }\end{array}$ \\
\hline 55806.51506 & 0.762 & $58.846 \pm 3.616$ & $58.846 \pm 3.616$ & $117.692 \pm 5.114$ & 0.01180 \\
\hline 55804.54459 & 0.471 & $58.846 \pm 3.616$ & $58.847 \pm 3.616$ & $117.693 \pm 5.114$ & 0.01221 \\
\hline 55811.74873 & 0.896 & $58.847 \pm 3.616$ & $58.846 \pm 3.616$ & $117.693 \pm 5.114$ & 0.01720 \\
\hline 55766.08239 & 0.745 & $58.848 \pm 3.616$ & $117.686 \pm 5.114$ & $176.534 \pm 6.264$ & 0.00989 \\
\hline 55830.23880 & 1.240 & $58.845 \pm 3.616$ & $176.538 \pm 6.264$ & $235.383 \pm 7.233$ & 0.01713 \\
\hline 55801.66075 & 1.066 & $117.702 \pm 5.114$ & $117.685 \pm 5.114$ & $235.386 \pm 7.233$ & 0.00827 \\
\hline 55794.32581 & 1.117 & $58.855 \pm 3.617$ & $176.532 \pm 6.263$ & $235.387 \pm 7.233$ & 0.00929 \\
\hline 55831.45798 & 1.880 & $117.700 \pm 5.114$ & $117.692 \pm 5.114$ & $235.392 \pm 7.233$ & 0.01391 \\
\hline 55822.77794 & 1.137 & $58.846 \pm 3.616$ & $176.546 \pm 6.264$ & $235.392 \pm 7.233$ & 0.01338 \\
\hline 55801.95704 & 2.098 & $58.846 \pm 3.616$ & $235.386 \pm 7.233$ & $294.233 \pm 8.086$ & 0.01416 \\
\hline 55804.71215 & 1.290 & $117.684 \pm 5.114$ & $176.549 \pm 6.264$ & $294.233 \pm 8.086$ & 0.01001 \\
\hline 55823.82889 & 1.051 & $58.846 \pm 3.616$ & $235.393 \pm 7.233$ & $294.240 \pm 8.086$ & 0.01032 \\
\hline 55822.73912 & 1.633 & $117.692 \pm 5.114$ & $235.384 \pm 7.233$ & $353.076 \pm 8.858$ & 0.01343 \\
\hline 55760.03465 & 0.632 & $58.848 \pm 3.616$ & $294.239 \pm 8.086$ & $353.087 \pm 8.858$ & 0.01071 \\
\hline 55801.75679 & 2.621 & $58.838 \pm 3.616$ & $294.251 \pm 8.087$ & $353.088 \pm 8.858$ & 0.01381 \\
\hline 55799.55611 & 1.312 & $117.702 \pm 5.114$ & $235.387 \pm 7.233$ & $353.089 \pm 8.858$ & 0.00642 \\
\hline 55780.13399 & 1.711 & $117.703 \pm 5.114$ & $235.389 \pm 7.233$ & $353.092 \pm 8.858$ & 0.01102 \\
\hline 55766.07149 & 0.848 & $117.704 \pm 5.114$ & $235.391 \pm 7.233$ & $353.094 \pm 8.858$ & 0.01050 \\
\hline 55768.37983 & 2.922 & $58.857 \pm 3.617$ & $294.255 \pm 8.087$ & $353.112 \pm 8.858$ & 0.01705 \\
\hline 55756.03029 & 1.168 & $117.687 \pm 5.114$ & $294.257 \pm 8.087$ & $411.944 \pm 9.568$ & 0.00935 \\
\hline 55756.56293 & 1.245 & $117.696 \pm 5.114$ & $294.248 \pm 8.086$ & $411.944 \pm 9.568$ & 0.01079 \\
\hline 55740.56791 & 2.973 & $117.696 \pm 5.114$ & $294.251 \pm 8.087$ & $411.947 \pm 9.568$ & 0.01556 \\
\hline 55831.96472 & 4.468 & $58.846 \pm 3.616$ & $411.929 \pm 9.568$ & $470.775 \pm 10.228$ & 0.03456 \\
\hline 55778.12332 & 2.165 & $117.694 \pm 5.114$ & $353.093 \pm 8.858$ & $470.787 \pm 10.229$ & 0.01238 \\
\hline 55776.73927 & 1.898 & $117.695 \pm 5.114$ & $353.093 \pm 8.858$ & $470.788 \pm 10.229$ & 0.00963 \\
\hline 55792.90704 & 6.883 & $117.694 \pm 5.114$ & $411.937 \pm 9.568$ & $529.631 \pm 10.849$ & 0.02718 \\
\hline 55779.79002 & 9.266 & $117.694 \pm 5.114$ & $411.940 \pm 9.568$ & $529.634 \pm 10.849$ & 0.04237 \\
\hline 55755.22928 & 3.861 & $176.552 \pm 6.264$ & $353.087 \pm 8.858$ & $529.640 \pm 10.849$ & 0.01210 \\
\hline 55772.93588 & 0.849 & $117.704 \pm 5.114$ & $411.941 \pm 9.568$ & $529.644 \pm 10.849$ & 0.01032 \\
\hline 55761.90026 & 1.219 & $117.704 \pm 5.114$ & $411.943 \pm 9.568$ & $529.648 \pm 10.849$ & 0.01217 \\
\hline 55755.87908 & 1.132 & $294.248 \pm 8.086$ & $235.400 \pm 7.233$ & $529.648 \pm 10.849$ & 0.00573 \\
\hline 55783.26307 & 0.793 & $58.856 \pm 3.617$ & $470.794 \pm 10.229$ & $529.650 \pm 10.849$ & 0.00322 \\
\hline 55762.79935 & 1.244 & $117.704 \pm 5.114$ & $411.952 \pm 9.568$ & $529.655 \pm 10.849$ & 0.01132 \\
\hline 55766.10282 & 2.214 & $294.247 \pm 8.086$ & $294.238 \pm 8.086$ & $588.485 \pm 11.436$ & 0.01453 \\
\hline 55792.98265 & 2.377 & $117.693 \pm 5.114$ & $470.793 \pm 10.229$ & $588.486 \pm 11.436$ & 0.01164 \\
\hline 55825.24424 & 2.172 & $117.700 \pm 5.114$ & $470.786 \pm 10.229$ & $588.486 \pm 11.436$ & 0.01260 \\
\hline 55810.05753 & 1.166 & $176.557 \pm 6.264$ & $411.933 \pm 9.568$ & $588.489 \pm 11.436$ & 0.01357 \\
\hline 55742.47985 & 1.287 & $176.545 \pm 6.264$ & $411.946 \pm 9.568$ & $588.490 \pm 11.436$ & 0.01300 \\
\hline 55747.50593 & 1.506 & $117.696 \pm 5.114$ & $470.802 \pm 10.229$ & $588.498 \pm 11.436$ & 0.01536 \\
\hline 55742.24282 & 3.543 & $294.241 \pm 8.086$ & $294.259 \pm 8.087$ & $588.500 \pm 11.436$ & 0.01447 \\
\hline 55829.60128 & 2.781 & $117.692 \pm 5.114$ & $529.629 \pm 10.849$ & $647.322 \pm 11.994$ & 0.01256 \\
\hline 55826.06293 & 3.057 & $176.546 \pm 6.264$ & $470.776 \pm 10.228$ & $647.323 \pm 11.994$ & 0.00379 \\
\hline 55792.31311 & 1.336 & $176.540 \pm 6.264$ & $470.793 \pm 10.229$ & $647.333 \pm 11.994$ & 0.00897 \\
\hline 55762.79186 & 3.297 & $117.686 \pm 5.114$ & $529.648 \pm 10.849$ & $647.334 \pm 11.994$ & 0.01830 \\
\hline 55779.32482 & 2.116 & $117.703 \pm 5.114$ & $529.635 \pm 10.849$ & $647.337 \pm 11.994$ & 0.01469 \\
\hline 55748.36688 & 1.896 & $235.392 \pm 7.233$ & $411.963 \pm 9.568$ & $647.355 \pm 11.994$ & 0.01075 \\
\hline 55776.23933 & 3.682 & $117.694 \pm 5.114$ & $588.491 \pm 11.436$ & $706.185 \pm 12.527$ & 0.00924 \\
\hline 55796.02792 & 3.817 & $117.703 \pm 5.114$ & $588.485 \pm 11.436$ & $706.188 \pm 12.527$ & 0.01597 \\
\hline 55765.69482 & 5.587 & $235.408 \pm 7.233$ & $470.789 \pm 10.229$ & $706.197 \pm 12.528$ & 0.01827 \\
\hline 55763.59627 & 1.525 & $117.704 \pm 5.114$ & $588.495 \pm 11.436$ & $706.199 \pm 12.528$ & 0.00532 \\
\hline 55811.84817 & 9.222 & $176.539 \pm 6.264$ & $588.481 \pm 11.436$ & $765.020 \pm 13.039$ & 0.02482 \\
\hline
\end{tabular}


TABLE 3 (CONTINUED)

\begin{tabular}{|c|c|c|c|c|c|}
\hline $\begin{array}{c}\text { Tmax } \\
(\text { BJD-2450000) }\end{array}$ & $\begin{array}{l}P \\
(\mathrm{~s})\end{array}$ & $\begin{array}{l}T_{r} \\
(\mathrm{~s})\end{array}$ & $\begin{array}{l}T_{d} \\
(\mathrm{~s})\end{array}$ & $\begin{array}{l}T_{t} \\
(\mathrm{~s})\end{array}$ & $\begin{array}{c}\text { Amplitude } \\
\text { (Relative Flux) }\end{array}$ \\
\hline 55808.84242 & 3.326 & $176.548 \pm 6.264$ & $588.472 \pm 11.436$ & $765.020 \pm 13.039$ & 0.01629 \\
\hline 55806.89444 & 2.641 & $294.241 \pm 8.086$ & $470.781 \pm 10.229$ & $765.022 \pm 13.039$ & 0.04012 \\
\hline 55810.38923 & 3.032 & $176.548 \pm 6.264$ & $588.481 \pm 11.436$ & $765.029 \pm 13.039$ & 0.00910 \\
\hline 55779.65108 & 6.755 & $176.551 \pm 6.264$ & $588.481 \pm 11.436$ & $765.031 \pm 13.039$ & 0.02747 \\
\hline 55777.26170 & 2.436 & $58.847 \pm 3.616$ & $706.185 \pm 12.527$ & $765.032 \pm 13.039$ & 0.01427 \\
\hline 55800.70583 & 1.053 & $176.549 \pm 6.264$ & $588.483 \pm 11.436$ & $765.032 \pm 13.039$ & 0.01235 \\
\hline 55773.50393 & 2.461 & $235.407 \pm 7.233$ & $529.627 \pm 10.849$ & $765.034 \pm 13.039$ & 0.04477 \\
\hline 55762.69582 & 2.510 & $58.839 \pm 3.616$ & $706.199 \pm 12.528$ & $765.038 \pm 13.039$ & 0.01041 \\
\hline 55748.38868 & 3.771 & $117.704 \pm 5.114$ & $647.337 \pm 11.994$ & $765.042 \pm 13.039$ & 0.01522 \\
\hline 55746.66405 & 1.236 & $117.704 \pm 5.114$ & $647.338 \pm 11.994$ & $765.043 \pm 13.039$ & 0.01060 \\
\hline 55743.15553 & 2.308 & $353.098 \pm 8.858$ & $411.946 \pm 9.568$ & $765.043 \pm 13.039$ & 0.01575 \\
\hline 55745.65597 & 2.618 & $117.705 \pm 5.114$ & $647.347 \pm 11.994$ & $765.052 \pm 13.039$ & 0.01126 \\
\hline 55821.60644 & 2.042 & $294.230 \pm 8.086$ & $529.632 \pm 10.849$ & $823.862 \pm 13.531$ & 0.00904 \\
\hline 55798.75171 & 4.518 & $58.838 \pm 3.616$ & $765.032 \pm 13.039$ & $823.871 \pm 13.531$ & 0.02078 \\
\hline 55794.34352 & 4.924 & $58.856 \pm 3.617$ & $765.026 \pm 13.039$ & $823.882 \pm 13.531$ & 0.01618 \\
\hline 55783.25353 & 3.925 & $235.388 \pm 7.233$ & $588.498 \pm 11.436$ & $823.886 \pm 13.531$ & 0.01514 \\
\hline 55763.58673 & 4.125 & $176.551 \pm 6.264$ & $647.342 \pm 11.994$ & $823.893 \pm 13.531$ & 0.01013 \\
\hline 55775.11139 & 3.272 & $117.704 \pm 5.114$ & $706.194 \pm 12.528$ & $823.897 \pm 13.531$ & 0.01310 \\
\hline 55740.48481 & 2.590 & $294.250 \pm 8.087$ & $529.652 \pm 10.849$ & $823.902 \pm 13.531$ & 0.01744 \\
\hline 55747.88464 & 3.972 & $58.857 \pm 3.617$ & $765.051 \pm 13.039$ & $823.908 \pm 13.531$ & 0.02227 \\
\hline 55820.70669 & 11.448 & $176.546 \pm 6.264$ & $706.171 \pm 12.527$ & $882.717 \pm 14.006$ & 0.02987 \\
\hline 55812.88346 & 5.447 & $58.855 \pm 3.617$ & $823.865 \pm 13.531$ & $882.720 \pm 14.006$ & 0.02041 \\
\hline 55792.81781 & 3.461 & $411.937 \pm 9.568$ & $470.783 \pm 10.229$ & $882.720 \pm 14.006$ & 0.01339 \\
\hline 55809.54465 & 3.030 & $117.693 \pm 5.114$ & $765.029 \pm 13.039$ & $882.722 \pm 14.006$ & 0.01444 \\
\hline 55807.14032 & 3.450 & $411.934 \pm 9.568$ & $470.789 \pm 10.229$ & $882.723 \pm 14.006$ & 0.01147 \\
\hline 55815.95868 & 2.639 & $58.856 \pm 3.617$ & $823.872 \pm 13.531$ & $882.728 \pm 14.006$ & 0.01146 \\
\hline 55744.32436 & 4.279 & $176.553 \pm 6.264$ & $706.196 \pm 12.528$ & $882.749 \pm 14.006$ & 0.01569 \\
\hline 55742.23055 & 5.768 & $117.705 \pm 5.114$ & $765.052 \pm 13.039$ & $882.757 \pm 14.006$ & 0.01393 \\
\hline 55817.51569 & 6.776 & $117.701 \pm 5.114$ & $823.864 \pm 13.531$ & $941.565 \pm 14.465$ & 0.02661 \\
\hline 55792.21979 & 1.686 & $176.541 \pm 6.264$ & $765.026 \pm 13.039$ & $941.567 \pm 14.465$ & 0.00954 \\
\hline 55806.74527 & 3.401 & $176.548 \pm 6.264$ & $765.021 \pm 13.039$ & $941.569 \pm 14.465$ & 0.01014 \\
\hline 55804.56094 & 2.295 & $176.539 \pm 6.264$ & $765.031 \pm 13.039$ & $941.571 \pm 14.465$ & 0.00944 \\
\hline 55743.93339 & 1.965 & $176.553 \pm 6.264$ & $765.043 \pm 13.039$ & $941.597 \pm 14.466$ & 0.00956 \\
\hline 55818.95147 & 5.622 & $58.846 \pm 3.616$ & $941.564 \pm 14.465$ & $1000.410 \pm 14.911$ & 0.02512 \\
\hline 55820.43834 & 4.285 & $117.701 \pm 5.114$ & $882.718 \pm 14.006$ & $1000.419 \pm 14.911$ & 0.00997 \\
\hline 55800.59413 & 2.617 & $117.702 \pm 5.114$ & $882.725 \pm 14.006$ & $1000.427 \pm 14.911$ & 0.01204 \\
\hline 55773.99094 & 2.775 & $58.848 \pm 3.616$ & $941.585 \pm 14.465$ & $1000.433 \pm 14.911$ & 0.01211 \\
\hline 55760.96984 & 3.584 & $117.696 \pm 5.114$ & $882.743 \pm 14.006$ & $1000.439 \pm 14.911$ & 0.01461 \\
\hline 55777.94827 & 9.265 & $176.542 \pm 6.264$ & $823.897 \pm 13.531$ & $1000.439 \pm 14.911$ & 0.02508 \\
\hline 55756.66238 & 4.502 & $176.552 \pm 6.264$ & $823.888 \pm 13.531$ & $1000.440 \pm 14.911$ & 0.01090 \\
\hline 55755.86341 & 3.832 & $176.552 \pm 6.264$ & $823.897 \pm 13.531$ & $1000.449 \pm 14.911$ & 0.00772 \\
\hline 55752.17713 & 3.504 & $176.553 \pm 6.264$ & $823.897 \pm 13.531$ & $1000.451 \pm 14.911$ & 0.01063 \\
\hline 55803.99153 & 3.654 & $294.233 \pm 8.086$ & $765.022 \pm 13.039$ & $1059.254 \pm 15.343$ & 0.01084 \\
\hline 55826.07315 & 3.786 & $176.546 \pm 6.264$ & $882.715 \pm 14.006$ & $1059.261 \pm 15.343$ & 0.00801 \\
\hline 55741.05083 & 10.207 & $58.849 \pm 3.616$ & $1000.455 \pm 14.911$ & $1059.304 \pm 15.343$ & 0.03355 \\
\hline 55805.48113 & 6.780 & $235.386 \pm 7.233$ & $882.724 \pm 14.006$ & $1118.109 \pm 15.763$ & 0.01629 \\
\hline 55792.62642 & 8.534 & $353.090 \pm 8.858$ & $765.027 \pm 13.039$ & $1118.117 \pm 15.763$ & 0.02378 \\
\hline 55751.93397 & 6.862 & $117.696 \pm 5.114$ & $1000.442 \pm 14.911$ & $1118.138 \pm 15.763$ & 0.03174 \\
\hline 55765.32020 & 4.999 & $176.551 \pm 6.264$ & $941.589 \pm 14.466$ & $1118.140 \pm 15.763$ & 0.01279 \\
\hline 55807.98013 & 4.321 & $117.702 \pm 5.114$ & $1059.261 \pm 15.343$ & $1176.963 \pm 16.173$ & 0.01249 \\
\hline 55792.84574 & 5.480 & $176.549 \pm 6.264$ & $1000.423 \pm 14.911$ & $1176.972 \pm 16.173$ & 0.02244 \\
\hline 55804.36682 & 5.572 & $235.386 \pm 7.233$ & $1000.417 \pm 14.911$ & $1235.803 \pm 16.572$ & 0.01366 \\
\hline 55818.06535 & 18.899 & $117.692 \pm 5.114$ & $1118.111 \pm 15.763$ & $1235.803 \pm 16.572$ & 0.04024 \\
\hline 55763.74271 & 5.420 & $235.399 \pm 7.233$ & $1000.445 \pm 14.911$ & $1235.845 \pm 16.572$ & 0.01580 \\
\hline
\end{tabular}


TABLE 3 (CONTINUED)

\begin{tabular}{|c|c|c|c|c|c|}
\hline $\begin{array}{c}\text { Tmax } \\
\text { (BJD-2450000) }\end{array}$ & $\begin{array}{l}P \\
(\mathrm{~s})\end{array}$ & $\begin{array}{l}T_{r} \\
(\mathrm{~s})\end{array}$ & $\begin{array}{l}T_{d} \\
(\mathrm{~s})\end{array}$ & $\begin{array}{l}T_{t} \\
(\mathrm{~s})\end{array}$ & $\begin{array}{c}\text { Amplitude } \\
\text { (Relative Flux) }\end{array}$ \\
\hline 55808.48143 & 6.886 & $294.241 \pm 8.086$ & $1000.415 \pm 14.911$ & $1294.656 \pm 16.962$ & 0.01014 \\
\hline 55752.86099 & 2.487 & $470.793 \pm 10.229$ & $823.897 \pm 13.531$ & $1294.690 \pm 16.962$ & 0.01033 \\
\hline 55748.43023 & 6.680 & $176.536 \pm 6.264$ & $1118.157 \pm 15.764$ & $1294.693 \pm 16.962$ & 0.01774 \\
\hline 55757.40209 & 13.431 & $235.400 \pm 7.233$ & $1059.296 \pm 15.343$ & $1294.696 \pm 16.962$ & 0.02852 \\
\hline 55740.24641 & 5.290 & $176.554 \pm 6.264$ & $1118.152 \pm 15.764$ & $1294.706 \pm 16.962$ & 0.01038 \\
\hline 55755.23405 & 7.243 & $58.857 \pm 3.617$ & $1235.850 \pm 16.572$ & $1294.707 \pm 16.962$ & 0.02040 \\
\hline 55799.53567 & 5.955 & $58.847 \pm 3.616$ & $1294.670 \pm 16.962$ & $1353.517 \pm 17.343$ & 0.02091 \\
\hline 55759.69340 & 8.248 & $235.400 \pm 7.233$ & $1118.134 \pm 15.763$ & $1353.535 \pm 17.344$ & 0.01900 \\
\hline 55748.56237 & 5.839 & $235.400 \pm 7.233$ & $1118.140 \pm 15.763$ & $1353.540 \pm 17.344$ & 0.01245 \\
\hline 55746.72876 & 6.982 & $294.240 \pm 8.086$ & $1059.301 \pm 15.343$ & $1353.542 \pm 17.344$ & 0.01395 \\
\hline 55759.69340 & 2.293 & $235.391 \pm 7.233$ & $1118.152 \pm 15.764$ & $1353.542 \pm 17.344$ & 0.00198 \\
\hline 55820.42199 & 10.170 & $176.539 \pm 6.264$ & $1235.811 \pm 16.572$ & $1412.350 \pm 17.716$ & 0.01346 \\
\hline 55797.44873 & 4.053 & $235.396 \pm 7.233$ & $1176.969 \pm 16.173$ & $1412.365 \pm 17.716$ & 0.01008 \\
\hline 55807.03611 & 20.538 & $294.241 \pm 8.086$ & $1176.964 \pm 16.173$ & $1471.205 \pm 18.082$ & 0.03700 \\
\hline 55791.63812 & 11.512 & $294.243 \pm 8.086$ & $1176.973 \pm 16.173$ & $1471.216 \pm 18.082$ & 0.02267 \\
\hline 55746.15729 & 4.354 & $117.697 \pm 5.114$ & $1353.550 \pm 17.344$ & $1471.247 \pm 18.082$ & 0.01144 \\
\hline 55806.00150 & 11.359 & $176.540 \pm 6.264$ & $1412.367 \pm 17.716$ & $1588.907 \pm 18.791$ & 0.02162 \\
\hline 55787.89945 & 7.928 & $58.847 \pm 3.616$ & $1530.075 \pm 18.440$ & $1588.922 \pm 18.791$ & 0.00838 \\
\hline 55768.84435 & 5.401 & $235.391 \pm 7.233$ & $1353.537 \pm 17.344$ & $1588.928 \pm 18.791$ & 0.01316 \\
\hline 55833.09059 & 25.717 & $117.700 \pm 5.114$ & $1530.033 \pm 18.440$ & $1647.734 \pm 19.136$ & 0.07710 \\
\hline 55819.06454 & 16.793 & $588.478 \pm 11.436$ & $1059.265 \pm 15.343$ & $1647.743 \pm 19.136$ & 0.02017 \\
\hline 55766.87590 & 18.093 & $235.391 \pm 7.233$ & $1412.386 \pm 17.717$ & $1647.777 \pm 19.136$ & 0.03545 \\
\hline 55773.95416 & 4.012 & $176.543 \pm 6.264$ & $1471.237 \pm 18.082$ & $1647.780 \pm 19.136$ & 0.01109 \\
\hline 55790.05588 & 38.271 & $411.947 \pm 9.568$ & $1294.676 \pm 16.962$ & $1706.623 \pm 19.475$ & 0.16402 \\
\hline 55816.58802 & 7.120 & $176.547 \pm 6.264$ & $1588.891 \pm 18.791$ & $1765.438 \pm 19.807$ & 0.01858 \\
\hline 55793.88104 & 24.987 & $117.702 \pm 5.114$ & $1647.764 \pm 19.136$ & $1765.466 \pm 19.808$ & 0.11313 \\
\hline 55826.02002 & 6.115 & $117.691 \pm 5.114$ & $1706.585 \pm 19.475$ & $1824.276 \pm 20.135$ & 0.01318 \\
\hline 55827.89170 & 10.892 & $117.691 \pm 5.114$ & $1765.429 \pm 19.807$ & $1883.121 \pm 20.457$ & 0.02212 \\
\hline 55819.08497 & 46.875 & $176.547 \pm 6.264$ & $1765.435 \pm 19.807$ & $1941.983 \pm 20.774$ & 0.14180 \\
\hline 55780.39486 & 10.869 & $588.489 \pm 11.436$ & $1471.224 \pm 18.082$ & $2059.714 \pm 21.395$ & 0.01187 \\
\hline 55765.45575 & 21.704 & $176.544 \pm 6.264$ & $1883.185 \pm 20.457$ & $2059.728 \pm 21.395$ & 0.04175 \\
\hline 55822.75342 & 10.331 & $353.085 \pm 8.858$ & $2000.826 \pm 21.087$ & $2353.911 \pm 22.872$ & 0.01707 \\
\hline 55828.63411 & 32.706 & $117.700 \pm 5.114$ & $2412.751 \pm 23.156$ & $2530.451 \pm 23.714$ & 0.04817 \\
\hline 55821.43071 & 23.129 & $294.240 \pm 8.086$ & $2589.304 \pm 23.988$ & $2883.544 \pm 25.314$ & 0.01867 \\
\hline 55756.89805 & 22.867 & $117.704 \pm 5.114$ & $2765.928 \pm 24.793$ & $2883.633 \pm 25.315$ & 0.03313 \\
\hline 55763.10313 & 119.328 & $117.704 \pm 5.114$ & $2942.472 \pm 25.572$ & $3060.177 \pm 26.078$ & 1.31184 \\
\hline 55764.80868 & 19.131 & $1059.301 \pm 15.343$ & $2059.729 \pm 21.395$ & $3119.030 \pm 26.328$ & 0.01630 \\
\hline 55810.14130 & 28.083 & $176.548 \pm 6.264$ & $3118.961 \pm 26.327$ & $3295.509 \pm 27.062$ & 0.02015 \\
\hline 55795.62606 & 59.471 & $117.703 \pm 5.114$ & $3236.660 \pm 26.820$ & $3354.362 \pm 27.303$ & 0.05526 \\
\hline 55822.11727 & 32.769 & $235.392 \pm 7.233$ & $3177.782 \pm 26.575$ & $3413.175 \pm 27.541$ & 0.02822 \\
\hline 55795.07436 & 37.409 & $353.089 \pm 8.858$ & $3589.768 \pm 28.245$ & $3942.857 \pm 29.601$ & 0.04263 \\
\hline 55792.70202 & 36.928 & $176.541 \pm 6.264$ & $3766.321 \pm 28.931$ & $3942.862 \pm 29.601$ & 0.04881 \\
\hline 55766.71856 & 117.197 & $235.391 \pm 7.233$ & $4119.463 \pm 30.257$ & $4354.854 \pm 31.109$ & 0.11637 \\
\hline 55741.94107 & 52.968 & $1059.303 \pm 15.343$ & $4296.050 \pm 30.899$ & $5355.353 \pm 34.498$ & 0.02498 \\
\hline
\end{tabular}

the $N_{2}$ frequency is a unitless parameter, because the unit of $\Sigma D T$ is time as well as the unit of $\Sigma D T$. However, the $N_{2}$ frequency is given in units of second/hour to facilitate the reading of the manuscript.

\section{RESULTS AND DISCUSSION}

We analysed the light curve and $(O-C)$ residuals of the system for the first time in the literature. The mass ratio of the system $(q)$ was found to be $0.743 \pm 0.001$, while the inclination $(i)$ was found to be $75^{\circ} .89 \pm 0^{\circ} .03$. This inclination $(i)$ value is in agreement with the inclination $(i)$ value of $72^{\circ} .47$ given by Coughlin et al. (2011). The mass of the primary component was found to be $0.64 M_{\odot}$ with a radius of $0.70 R_{\odot}$, while it was found to be $0.57 M_{\odot}$ for the secondary component with a radius of $0.65 R_{\odot}$. Although these parameters are a bit larger than those found by Coughlin et al. (2011), 


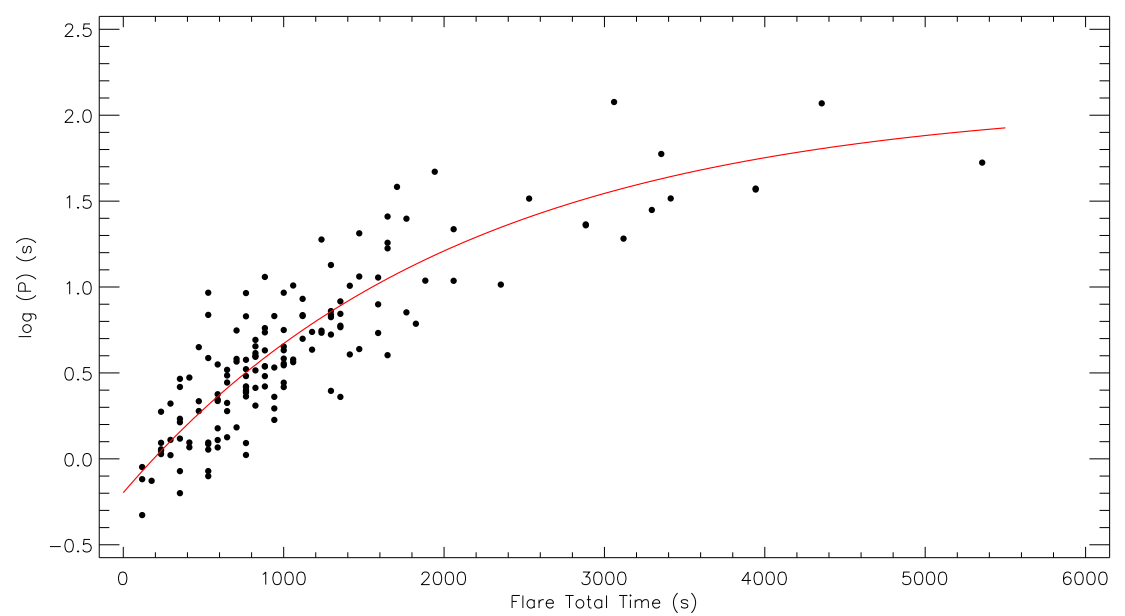

Fig. 6. The OPEA model derived from 149 flares detected in the available short cadence data of KIC 12004834 is shown. In the figure, the filled circles represent the calculated $\log (P)$ values from observations, while the red line represents the OPEA model. The color figure can be viewed online.

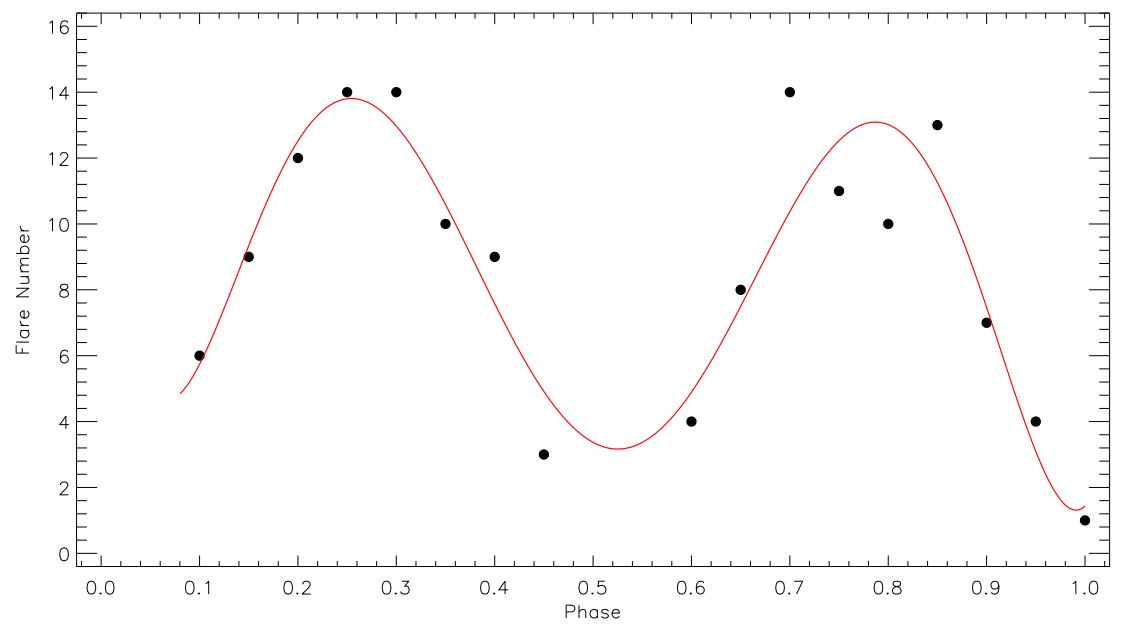

Fig. 7. The distribution of flare total number in each phase interval of 0.05, plotted versus phase for 149 flares. The color figure can be viewed online.

it can be assumed that they are in agreement. In addition, these values indicate that KIC 12004834 is a simple close binary system. Indeed, the possible semi-major axis was found to be as small as $1.84 R_{\odot}(\approx 0.0086 \mathrm{AU})$, which indicates that there must be a distance of $0.49 R_{\odot}$ between the surfaces of the components. In this case, it is possible that each component can trigger the magnetic activity of the other.

If being in a very close binary affects the chromospheric patterns of the active component, we should see an effect: an increase of the flare frequency or of the Plateau value. KIC 12004834 was continuously observed over 89.19 days from JD 2455739.83568694 to JD 24 55833.27789066, and we detected 149 flare events. The flare frequency $N_{1}$, the general flare number per an hour, was found to be $0.070 h^{-1}$, while the flare frequency $N_{2}$, the averaged flare energy per an hour, was computed as 0.62 second/hour. However, according to these results, KIC 12004834 did not show a high magnetic activity, not at the expected level.

Comparing the target to similar systems, we can easily notice that like KIC 12004834, both FL Lyr and KIC 9761199 are binary systems, but with high level chromospheric activity. Indeed, the flare frequencies of FL Lyr were recently found to be $N_{1}=0.41632 h^{-1}$ and $N_{2}=0.00027$ by (Yoldaş \& Dal 2016). However, the radii of the FL Lyr components were given as $R_{1}=1.283 R_{\odot}, R_{2}=0.963 R_{\odot}$ 
TABLE 4

THE OPEA MODEL PARAMETERS DERIVED BY USING THE LEAST-SQUARES METHOD

\begin{tabular}{lr}
\hline Parameter & \multicolumn{1}{c}{ Value } \\
\hline$Y_{0}$ & $-0.197 \pm 0.081$ \\
Plateau & $2.093 \pm 0.236$ \\
$K$ & $0.00048 \pm 0.00010$ \\
Tau & 2098.68 \\
Half - life & 1454.7 \\
$95 \%$ Confidence Intervals & \\
\hline$Y_{0}$ & -0.355 to -0.038 \\
Plateau & 1.630 to 2.556 \\
$K$ & 0.00029 to 0.00067 \\
Tau & 1497.65 to 3505.49 \\
Half - life & 1038.09 to 2429.82 \\
Goodness of Fit & \\
\hline$R^{2}$ & 0.7628 \\
$p-$ value (D'Agostino-Pearson) & 0.0001 \\
$p-$ value (Shapiro-Wilk) & 0.0001 \\
$p-$ value (Kolmogorov-Smirnov) & 0.0005 \\
\hline
\end{tabular}

with a semi-major axis of $a=9.17 R_{\odot}$ by Eker et al. (2014). In the case of KIC 9761199, Yoldas \& Dal (2017) found the Plateau value as $1.951 \mathrm{~s}$, while the flare frequencies, $N_{1}$ and $N_{2}$, were found to be $0.01351 h^{-1}$ and 0.00006 , respectively. The authors computed the masses of the primary and secondary components as $0.57 M_{\odot}, 0.39 M_{\odot}$, and the radii of the components as $0.62 R_{\odot}$ and $0.56 R_{\odot}$ with a semimajor axis of $a=5.16 R_{\odot}$. According to these results, if being a close binary affects the surface magnetic activity, KIC 12004834 should exhibit more frequent or more powerful flares than FL Lyr. On the other hand, according to Dal \& Evren (2011); Dal (2012), it is a controversial topic whether the flare frequencies are an indicator of flare activity level, or not. There is one more parameter that may be taken an indicator for the flare activity level, which is the Plateau value. The Plateau value was found to be $2.093 \pm 0.236 \mathrm{~s}$ from the OPEA model of KIC 12004834. However, Yoldaş \& Dal (2016) found the Plateau value as $1.232 \pm 0.069 \mathrm{~s}$ for FL Lyr. According to these results, the activity level of KIC 12004834 is nearly two times higher than FL Lyr, as expected. With an increasing number of eclipsing binaries with a flaring component, we may determine which parameter is real indicator for the flare activity level.

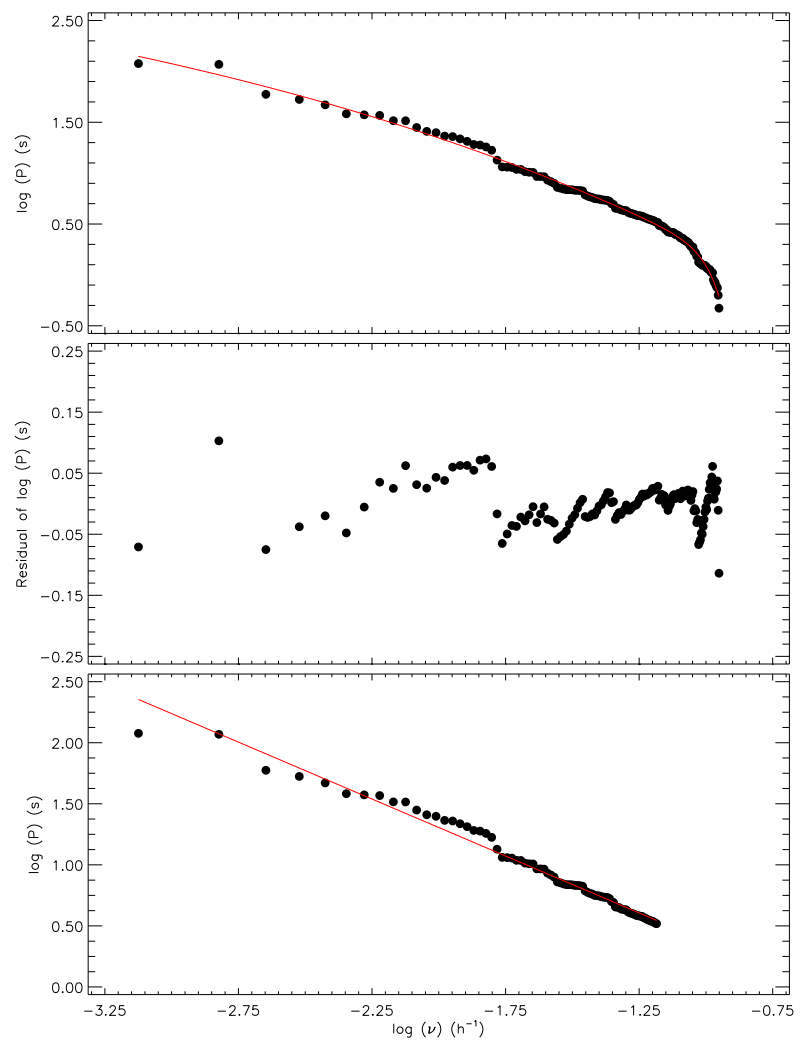

Fig. 8. Cumulative flare frequencies and model computed for 149 flares obtained from KIC 12004834. In the upper panel, the variation of the flare equivalent durations versus the cumulative flare frequency is shown, while the residuals obtained from the model are shown in the middle panel. The bottom panel shows the linear part of the flare energy spectrum and its linear representation. The color figure can be viewed online.

Using the regression calculations, the half-life value was found to be $1454.7 \mathrm{~s}$ from the OPEA model for KIC 12004834. In the case of FL Lyr, it is $2291.7 \mathrm{~s}$ (Yoldas \& Dal 2016). This means that a flare occurring on FL Lyr can reach the maximum energy level when the flare total duration reaches 38 minutes, while it takes 24.245 minutes for KIC 12004834. Moreover, the maximum flare rise time $\left(T_{r}\right)$ obtained from the flares of KIC 12004834 was found to be $1059.303 \mathrm{~s}$, while the maximum flare total time $\left(T_{t}\right)$ was found to be $5355.050 \mathrm{~s}$. However, these values are $T_{r}=5179.00 \mathrm{~s}$ and $T_{t}=12770.62 \mathrm{~s}$ for FL Lyr. As a result, the FL Lyr flare time scales are obviously larger than those obtained from KIC 12004834, which is in agreement with the results found by Dal \& Evren (2011); Dal (2012) for single flare stars of type dMe. 
However, there is one more controversial feature of KIC 12004834, which is stellar spot activity. During the analysis process, we recognised that the synthetic curve derived without any stellar spot does not fit the observations around phase 0.27 . Because of this, the results of the light curve analysis point to the presence of stellar spot activity on one of the components. Considering the temperatures of the components we assumed that the flare activity is a sinusoidal variation caused by the rotational modulation due to the stellar cool spots. Indeed, this sinusoidal variation could be easily modelled with a cool spot on the primary component as seen in the 3D model of Roche geometry shown in Figure 4. However, the analyses indicated that the location of the spot is not changed on the primary component along 89.19 day despite the presence of high level flare activity. Considering the rapid variation in the flare behaviour, a stable spotted area on the component is very interesting. According to Hall et al. (1989) and Gershberg (2005), it is well known that the spotted areas on the active components of some RS CVn binaries can keep their shapes and locations for as long as two years. Therefore, the behavior of the cool spot activity observed for KIC 12004834 is not inconsistent with the stellar spot activity phenomenon.

In addition, considering the distribution of the total flare number in each phase interval of 0.05 , it is noticed that the flares tend to occur in two specific phases, 0.25 and 0.75 , as seen in Figure 7. Since the spotted area is seen around phase 0.27 , the behavior of the flare activity of this close binary system KIC 12004834 can be understood.

At this point, one can question whether the sinusoidal variation out-of-eclipses is really caused by the stellar spots. In the literature, Tran et al. (2013) found a way to obtain the sign of the spot activity. They demonstrated that the spot activity remarkably affects the variations of the $(O-C)$ residuals, especially for the $(O-C)_{I I}$ residuals. Tran et al. (2013) reported that the stellar spot activity occurring on the active component causes the $(O-C)_{I I}$ residuals of both the primary and secondary minima to vary synchronously in a sinusoidal manner, but in opposite directions. We could not determine the minima times from the Kepler long cadence data because the orbital period of KIC 12004834 is short, $0^{\mathrm{d}} .262317$. However, we determined all the minima times from the available short cadence data. In the Kepler Mission Database, the short cadence data of observations are covered over 100 days. Because of this, the sinusoidal variation cannot be properly seen. On the other hand, we determined that the minima times computed from the primary and the secondary minima are separated from each other. This is enough evidence for the spot presence on one component.

We wish to thank the Turkish Scientific and Technical Research Council for supporting this work through grant No. 116F213. We thank the referee for useful comments that have contributed to the improvement of the paper. We also thank Dr. O. Özdarcan for the useful scripts that have supported us, providing an easy way to solve hard calculations.

\section{REFERENCES}

Armstrong, D. J., Gómez Maqueo Chew, Y., Faedi, F., \& Pollacco, D. 2014, MNRAS, 437, 3473

Balona, L. A. 2015, MNRAS, 447, 2714

Benz, A. O. 2008, LRSP, 5, 1

Borucki, W. J., Koch, D., Basri, G., et al. 2010, Sci, 327, 977

Caldwell, D. A., Kolodziejczak, J. J., \& Van Cleve, J. E. 2010, ApJL, 713, L92

Carrington, R. C. 1859, MNRAS, 20, 13

Coughlin, J. L., López-Morales, M., Harrison, T. E., Ule, N., \& Hoffman, D. I. 2011, AJ, 141, 78

Cutri, R. M., Skrutskie, M. F., van Dyk, S., et al. 2003, The IRSA 2MASS all sky point source catalog. NASA/IPAC Infrared Science Archive http: //irsa.ipac.caltech.edu/applications/Gator

D'Agostino, R. B. \& Stephens, M. A. 1986, GoodnessOf-Fit Techniques, ed. Ralph B. D'Agostino \& M. A. Stephens (New York, NY: Dekker)

Dal, H. A. 2012, PASJ, 64, 82

Dal, H. A. \& Evren, S. 2010, AJ, 140, 483 2011, AJ, 141, 33

Dawson, B. \& Trapp, R. G. 2004, Basic and Clinical Biostatistics, (New York, NY: McGraw-Hill)

Debosscher, J., Blomme, J., Aerts, C., \& De Ridder, J. 2011, A\&A, 529, 89

Eker, Z., Bilir, S., Soydugan, F., et al. 2014, PASA, 31, 24

Gershberg, R. E. 2005, Solar-Type Activity in MainSecauence Stars, (New York, NY: Springer)

Gershberg, R. E. \& Shakhovskaya, N. I. 1983, Ap\&SS, 95, 235

Gershberg, R. E. 1972, Ap\&SS, 19, 75

Green, S. B., Salkind, N. J. \& Akey, T. M. 1999, Using SPSS for Windows: Analyzing and Understanding Data (New Jersey, NJ: Perarson)

Haisch, B., Strong, K. T., \& Rodonó, M. 1991, ARA\&A, 29, 275

Hall D. S., Henry G. W., \& Sowell J. R. 1989, AJ, 99, 396 
Hodgson, R. 1859, MNRAS, 20, 15

Ishida, K., Ichimura, K., Shimizu, Y. \& Mahasenaputra. 1991, Ap\&SS, 182, 227

Jenkins, J. M., Caldwell, D. A., Chandrasekaran, H., et al. 2010a, ApJL, 713, L87

Jenkins, J. M., Chandrasekaran, H., McCauliff, S. D., et al. 2010b, SPIE, 7740, 77400

Kwee, K. K. \& van Woerden, H. 1956, BAN, 12, 327

Koch, D. G., Borucki, W. J., Basri, G., et al. 2010, ApJL, 713, L79

Lucy, L. B. 1967, ZA, 65, 89

Matijevič, G., Prša, A., Orosz, J. A., et al. 2012, AJ, 143, 123

Murphy, S. J. 2012, MNRAS, 422, 665

Murphy, S. J., Shibahashi, H., \& Kurtz, D. W. 2013, MNRAS, 430, 2986

Özdarcan, O., Yoldaş, E., \& Dal, H. A. 2018, RMxAA, 54,37

Prša, A. \& Zwitter, T. 2005, ApJ, 628, 426
Rodonó, M. 1986, NASSP, 492, 409

Rucinski, S. M. 1969, AcA, 19, 245

Slawson, R., Prša, A., Welsh, W. F., et al. 2011, AJ, 142, 160

Taylor, J. 1997, Introduction to Error Analysis, the Study of Uncertainties in Physical Measurements, (2nd ed.; Sausalito, CA: University Science Books)

Tokunaga, A. T. 2000, in Allen's Astrophysical Quantities, ed. A. N. Cox (Springer)

Tran, K., Levine, A., Rappaport, S., et al. 2013, ApJ, 774,81

Van Hamme, W. 1993, AJ, 106, 2096

Walkowicz, L. M. \& Basri, G. S. 2013, MNRAS, 436, 1883

Watson, C. L. 2006, SASS, 25, 47

Wilson, R. E. 1990, ApJ, 356, 613

Wilson, R. E. \& Devinney, E. J. 1971, ApJ, 166, 605

Yoldaş, E. \& Dal, H. A. 2016, PASA, 33,16

. 2017, RMxAA, 53, 67

H. A. Dal and E. Yoldaş: Department of Astronomy and Space Sciences, University of Ege, Bornova, 35100 İzmir, Turkey (ezgiyoldas@gmail.com). 